\title{
Polynomial Approximation of Divergence-Free Functions
}

\author{
By Giovanni Sacchi Landriani and Hervé Vandeven
}

\begin{abstract}
We study the best approximation of a divergence-free function by a divergence-free algebraic or trigonometric polynomial and we prove an optimal estimate. In a particular case we give also an optimal result for the polynomial approximation of a function and its divergence.
\end{abstract}

1. Introduction. In this paper we study the problem of the approximation of a divergence-free function by divergence-free polynomials. This problem is closely related to the analysis of the convergence of spectral methods for the incompressible Navier-Stokes equations in a cube with appropriate boundary conditions: Dirichlet in all the directions, or Dirichlet in one direction and periodic in the others. In these methods the discrete solution is an algebraic (or a trigonometric) polynomial which satisfies a discrete continuity equation. The convergence proofs are essentially based on the fact that the distance between the exact solution and the discrete solution (approximation error) is related to the distance between the exact solution and the space of divergence-free polynomials (projection error). This distance is in general associated with the $\mathbf{H}^{\mathbf{1}}$-norm (see, e.g., [8]), but also with the $\mathbf{H}^{2}$-norm, typically when tau-methods are considered (see, e.g., [11], [24]). Recently, C. Bernardi, C. Canuto and Y. Maday [5] have analyzed an approximation of the Stokes equations involving Chebyshev polynomials; the approximation then involves weighted norms.

For these reasons it appears useful to prove optimal convergence estimates in weighted norms for the projection error onto the space of divergence-free polynomials. Some results of this type were proven in [5] and [25] for weighted norms in a two-dimensional domain, in [8] for unweighted norms in a two-dimensional domain, and in [7] for unweighted norms in a two- or three-dimensional domain for a particular set of boundary conditions.

Some approximation results in Sobolev spaces for orthogonal polynomials can be found in [3], [6], [10], [14], [17], [18], [20], [22]; we shall use this kind of results for the analysis of the projection operator on the space of divergence-free polynomials.

An outline of the paper is as follows. In Section 2 we define some projection operators on the space of divergence-free functions which are polynomial only with respect to one variable. These operators are useful in studying the best approximation of a divergence-free function by divergence-free polynomials. In the case of unweighted norms, we also define a projection operator such that, given a function $\mathbf{u}$, the divergence of this projection of $\mathbf{u}$ is related to the $\mathbf{H}^{1}$-projection of the divergence of $\mathbf{u}$. This result can be useful in the analysis of problems where the

Received July 9, 1987.

1980 Mathematics Subject Classification (1985 Revision). Primary 41A10, 35Q10, 65N35. 
divergence of the solution is a given function not identically zero. In Section 3 we consider the case of a function which is periodic in some directions, both for Cartesian and cylindrical coordinates. We present results also for these cases. These results can be used in the analysis of the methods presented in [13], [16], [21]. In Section 4 we provide an application of the preceding results; we analyze a spectral approximation of the Stokes problem in cylindrical coordinates and we prove an optimal convergence estimate. Finally, in the appendix we derive an interpolation result for the spaces of divergence-free functions; such a result is useful for the estimates of Section 3, where periodicity is assumed in some directions.

Notations. Let $\Delta$ be a set in $\mathbb{R}, \mathbb{R}^{2}$ or $\mathbb{R}^{3}$. For any nonnegative integer $N$ we denote by $\mathbf{P}_{N}(\Delta)$ the space of all polynomials on $\Delta$ of degree $\leq N$ in each variable.

Let $\Theta$ be the interval $(0,2 \pi)$. For any integer $d \geq 1$, and for any nonnegative integer $K$, we denote by $\mathbf{S}_{K}\left(\Theta^{d}\right)$ the space of trigonometric polynomials on $\Theta^{d}$ of degree $\leq K$.

For any function $\varphi: \mathbb{R} \rightarrow \mathbb{R}$ and for any nonnegative integer $j$ we denote by $\varphi^{(j)}$ the derivative of $\varphi$ of order $j$.

For any $\mathbf{k}=\left(k_{1}, \ldots, k_{d}\right) \in \mathbb{Z}^{d}$ we set

$$
|\mathbf{k}|_{1}=\sum_{j=1}^{d}\left|k_{j}\right| \text { and }|\mathbf{k}|_{2}=\left(\sum_{j=1}^{d} k_{j}^{2}\right)^{1 / 2} .
$$

For any real number $s$ we denote by $[s]$ its integral part.

Given a function space $\mathbf{V}$ and a nonnegative integer $d$, we denote by $\mathbf{V}^{d}$ the corresponding vector-valued function space; the norm in $\mathbf{V}^{d}$ is denoted by the same symbol as the norm in $\mathbf{V}$. If $P$ is an operator on $\mathbf{V}$, we denote by $\mathbf{P}$ the operator on $\mathbf{V}^{d}$ defined by

$$
\forall \mathbf{v}=\left(v_{1}, \ldots, v_{d}\right) \in \mathbf{V}^{d}, \quad \forall j, 1 \leq j \leq d,(\mathbf{P v})_{j}=P v_{j}
$$

The norm of a Banach space $\mathbf{Y}$ is denoted by $\|\cdot\|_{Y}$.

2. Projection Operators for Homogeneous Boundary Conditions. In this section, $\Omega$ is the domain $\Lambda^{3}$ in $\mathbb{R}^{3}$, with $\Lambda=(-1,1)$. The generic point in $\Omega$ is denoted by $\mathbf{x}=(x, y, z)$.

Let $\rho: \Lambda \rightarrow \mathbf{R}$ be either the Legendre weight function defined by $\rho(\zeta)=1$ or the Chebyshev weight function defined by $\rho(\zeta)=\left(1-\varsigma^{2}\right)^{-1 / 2}$. We define a weight function on $\Omega$ by $\omega(\mathbf{x})=\rho(x) \rho(y) \rho(z)$. When $\rho$ and $\omega$ are the Legendre (resp. Chebyshev) weights, we set $\sigma_{0}=1 / 2\left(\right.$ resp. $\left.\sigma_{0}=1 / 4\right)$.

2.1. The Weighted Sobolev Spaces. We recall here briefly some definitions of weighted Sobolev spaces (see, e.g., [1], [2], [15]). Let

$$
\mathbf{L}_{\rho}^{2}(\Lambda)=\left\{\varphi: \Lambda \rightarrow \mathbf{R} ; \varphi \text { is measurable and } \int_{\Lambda} \varphi^{2}(\varsigma) \rho(\varsigma) d \varsigma<+\infty\right\}
$$

be the Lebesgue space associated with the measure $\rho(\varsigma) d \varsigma$, provided with the inner product

$$
(\varphi, \psi)_{\rho}=\int_{\Lambda} \varphi(\varsigma) \psi(\varsigma) \rho(\varsigma) d \varsigma
$$

and the norm $\|\cdot\|_{0, \rho}=(\cdot, \cdot)_{\rho}^{1 / 2}$. 
A scale of weighted Sobolev spaces is defined as follows: for any integer $m \geq 0$, $\mathbf{H}_{\rho}^{m}(\Lambda)$ is the subspace of $\mathrm{L}_{\rho}^{2}(\Lambda)$ of the functions such that their distributional derivatives of order $\leq m$ all belong to $\mathbf{L}_{\rho}^{2}(\Lambda)$; it is a Hilbert space for the inner product associated with the norm

$$
\|\varphi\|_{m, \rho}=\left(\sum_{k=0}^{m}\left\|\varphi^{(k)}\right\|_{0, \rho}^{2}\right)^{1 / 2} .
$$

For a real number $s=m+\alpha, 0<\alpha<1$, we define $\mathbf{H}_{\rho}^{s}(\Lambda)$ to be the interpolation space between $\mathbf{H}_{\rho}^{m+1}(\Lambda)$ and $\mathbf{H}_{\rho}^{m}(\Lambda)$ of index $1-\alpha$ (see [4], [15]); we denote its norm by $\|\cdot\|_{s, \omega}$. For any real number $s$ with $s>\sigma_{0}$, for any function $\varphi$ in $\mathbf{H}_{\rho}^{s}(\Lambda)$, and for any integer $j$ with $0 \leq j<s-\sigma_{0}$, the boundary values $\varphi^{(j)}( \pm 1)$ are well defined (see [15, Vol. 1, Ch. 1, Theorem 11.5] and [12, Theorem 7.1]).

For any real number $s$ we define $\mathbf{H}_{\rho, 0}^{s}(\Lambda)$ to be the closure in $\mathbf{H}_{\rho}^{s}(\Lambda)$ of the space $\mathbf{D}(\Lambda)$ of infinitely differentiable functions with compact support in $\Lambda$. For a real number $s$ such that $s \notin \mathbb{N}+\sigma_{0}$ and $s>\sigma_{0}$ we have (see [6])

$$
\mathbf{H}_{\rho, 0}^{s}(\Lambda)=\left\{\varphi \in \mathbf{H}_{\rho}^{s}(\Lambda) ; \forall j \in \mathbb{N}, 0 \leq j \leq\left[s-\sigma_{0}\right], \varphi^{(j)}(-1)=\varphi^{(j)}(1)=0\right\}
$$

Let now

$$
\mathbf{L}_{\omega}^{2}(\Omega)=\left\{u: \Omega \rightarrow \mathbb{R} ; u \text { is measurable and } \int_{\Omega} u^{2}(\mathbf{x}) \omega(\mathbf{x}) d \mathbf{x}<+\infty\right\}
$$

be the Lebesgue space associated with the measure $\omega(\mathbf{x}) d \mathbf{x}$, provided with the inner product

$$
(u, v)_{\omega}=\int_{\Omega} u(\mathbf{x}) v(\mathbf{x}) \omega(\mathbf{x}) d \mathbf{x}
$$

and the norm $\|\cdot\|_{0, \omega}=(\cdot, \cdot)_{\omega}^{1 / 2}$.

A scale of weighted Sobolev spaces is defined as follows: for any integer $m \geq 0$, $\mathbf{H}_{\omega}^{m}(\Omega)$ is the subspace of $\mathbf{L}_{\omega}^{2}(\Omega)$ of the functions such that their distributional derivatives of order $\leq m$ all belong to $\mathbf{L}_{\omega}^{2}(\Omega)$; it is a Hilbert space for the inner product associated with the norm

$$
\|u\|_{m, \omega}=\left(\sum_{\substack{\mathbf{k} \in N^{3} \\|\mathbf{k}|_{1} \leq m}} \int_{\Omega}\left|\frac{\partial^{k_{1}+k_{2}+k_{3}}}{\partial x^{k_{1}} \partial y^{k_{2}} \partial z^{k_{3}}} u(\mathbf{x})\right|^{2} \omega(\mathbf{x}) d \mathbf{x}\right)^{1 / 2} .
$$

For a real number $s=m+\alpha, 0<\alpha<1$, we define $\mathbf{H}_{\omega}^{s}(\Omega)$ to be the interpolation space between $\mathbf{H}_{\omega}^{m+1}(\Omega)$ and $\mathbf{H}_{\omega}^{m}(\Omega)$ of index $1-\alpha$; we denote its norm by $\|\cdot\|_{s, \omega}$.

For any real number $s$ we define $\mathbf{H}_{\omega, 0}^{s}(\Omega)$ to be the closure in $\mathbf{H}_{\omega}^{s}(\Omega)$ of the space $\mathbf{D}(\Omega)$ of infinitely differentiable functions with compact support in $\Omega$.

When we treat the case of the Legendre weight, the subscripts $\rho$ and $\omega$ will be deleted.

2.2. Projection Operators onto Divergence-Free Polynomials. We define a onedimensional projection operator that we shall use in the sequel. We recall the following result due to Y. Maday [17]. 
THEOREM 2.1. Let $r$ and $s$ be two real numbers such that $r \notin \mathbb{N}+\sigma_{0}$ and $0 \leq s \leq r$. For any integer $N$ with $N \geq 1$, there exists an operator $\Pi_{r, N}^{\circ}$ from $\mathbf{H}_{\rho}^{r}(\Lambda) \cap \mathbf{H}_{\rho, 0}^{s}(\Lambda)$ onto $\mathbf{P}_{N}(\Lambda) \cap \mathbf{H}_{\rho, 0}^{s}(\Lambda)$ which satisfies

$$
\forall \varphi \in \mathbf{H}_{\rho}^{\sigma}(\Lambda) \cap \mathbf{H}_{\rho, 0}^{s}(\Lambda), \quad\left\|\varphi-\Pi_{r, N}^{\circ s} \varphi\right\|_{\nu, \rho} \leq C N^{\nu-\sigma}\|\varphi\|_{\sigma, \rho},
$$

for any real numbers $\nu$ and $\sigma$ with $0 \leq \nu \leq r \leq \sigma$ and for a positive constant $C$ independent of both $\varphi$ and $N$.

For any real number $r$ not in $\mathbb{N}+\sigma_{0}$, with $r \geq 1$, we now define a polynomial $\Pi_{r, N} \varphi$ which coincides with $\varphi$ at the boundary with all the derivatives up to order $\left[r-\sigma_{0}\right]$. In the next corollary, which is a direct consequence of Theorem 2.1, we show that the operator $\Pi_{r, N}$ has the same asymptotic properties as $\Pi_{r, N}^{\circ r}$. Naturally, if $\varphi$ belongs to $\mathbf{H}_{\rho, 0}^{r}(\Lambda)$, then $\Pi_{r, N} \varphi$ is equal to $\Pi_{r, N}^{\circ r} \varphi$.

COROLlaRY 2.1. Let $r$ be a real number with $r \geq 1$ and $r \notin \mathbb{N}+\sigma_{0}$. For any integer $N$ with $N \geq 2\left[r-\sigma_{0}\right]+1$, there exists an operator $\Pi_{r, N}$ from $\mathbf{H}_{\rho}^{r}(\Lambda)$ onto $\mathbf{P}_{N}(\Lambda)$ which satisfies

$$
\forall \varphi \in \mathbf{H}_{\rho}^{r}(\Lambda), \forall j \in \mathbb{N}, 0 \leq j \leq\left[r-\sigma_{0}\right], \quad\left(\Pi_{r, N} \varphi\right)^{(j)}( \pm 1)=\varphi^{(j)}( \pm 1) .
$$

Moreover, for any real numbers $\nu$ and $\sigma$ such that $0 \leq \nu \leq r \leq \sigma$ there exists a positive constant $C$ independent of $N$ such that

$$
\forall \varphi \in \mathbf{H}_{\rho}^{\sigma}(\Lambda), \quad\left\|\varphi-\Pi_{r, N} \varphi\right\|_{\nu, \rho} \leq C N^{\nu-\sigma}\|\varphi\|_{\sigma, \rho}
$$
that

Proof. Let $\varphi$ be a function in $\mathbf{H}_{\rho}^{r}(\Lambda)$ and $q$ be the element of $P_{2\left\{r-\sigma_{0}\right]+1}(\Lambda)$ such

$$
\forall j \in \mathbb{N}, 0 \leq j \leq\left[r-\sigma_{0}\right], \quad \varphi^{(j)}( \pm 1)=q^{(j)}( \pm 1) .
$$

It follows from (2.1) that $\varphi-q$ belongs to $\mathbf{H}_{\rho, 0}^{r}(\Lambda)$. Set

$$
\Pi_{r, N} \varphi=q+\Pi_{r, N}^{\circ r}(\varphi-q) .
$$

From (2.5) and (2.6) we obtain (2.3).

Let now $\nu$ and $\sigma$ be two real numbers with $0 \leq \nu \leq r \leq \sigma$, and assume that $\varphi$ belongs to $\mathbf{H}_{\rho}^{\sigma}(\Lambda)$. We have

$$
\|q\|_{\sigma, \rho} \leq C \sum_{j=0}^{\left[r-\sigma_{0}\right]}\left\{\left|\varphi^{(j)}(-1)\right|+\left|\varphi^{(j)}(1)\right|\right\} .
$$

Let $\varepsilon=\left(r-\sigma_{0}\right)-\left[r-\sigma_{0}\right]$. Since $r$ is not an element of $\mathbb{N}+\sigma_{0}$, we have $\varepsilon>0$. Using the imbedding $\mathbf{H}_{\rho}^{\sigma_{0}+\varepsilon}(\Lambda) \subset \mathbf{L}^{\infty}(\Lambda)$, we deduce

$$
\|q\|_{\sigma, \rho} \leq C \sum_{j=0}^{\left[r-\sigma_{0}\right]}\left\|\varphi^{(j)}\right\|_{L^{\infty}(\Lambda)} \leq C\|\varphi\|_{r, \rho} \leq C\|\varphi\|_{\sigma, \rho} .
$$

Using (2.2) and (2.7), we get

$$
\begin{aligned}
\left\|\varphi-\Pi_{r, N} \varphi\right\|_{\nu, \rho} & =\left\|(\varphi-q)-\Pi_{r, N}^{\circ r}(\varphi-q)\right\|_{\nu, \rho} \\
& \leq C N^{\nu-\sigma}\|\varphi-q\|_{\sigma, \rho} \leq C^{\prime} N^{\nu-\sigma}\|\varphi\|_{\sigma, \rho} .
\end{aligned}
$$

This completes the proof of (2.4). 
We now define an operator with values in the space of functions which are polynomial with respect to one variable.

For any real number $r$ with $r \geq 1$, and for any function $u$ of $\mathbf{H}_{\omega}^{r}(\Omega)$, we set

$$
\left(\Pi_{r, N}^{z} u\right)(\mathbf{x})=\left(\Pi_{r, N} u(x, y, \cdot)\right)(z) \text { for a.e. }(x, y) \text { in } \Lambda^{2} \text {. }
$$

The operators $\Pi_{r, N}^{x}$ and $\Pi_{r, N}^{y}$ are defined in the same way. We remark that $\Pi_{r, N}^{z}$ commutes with the derivatives with respect to the $x$ and $y$ variables, i.e.,

$$
\frac{\partial}{\partial x} \Pi_{r, N}^{z} u=\Pi_{r, N}^{z} \frac{\partial u}{\partial x} \quad \text { and } \quad \frac{\partial}{\partial y} \Pi_{r, N}^{z} u=\Pi_{r, N}^{z} \frac{\partial u}{\partial y}
$$

provided that the derivatives of $u$ belong to the domain of $\Pi_{r, N}^{z}$.

If $\mathbf{u}$ is a divergence-free function, we want to study the best approximation of $\mathbf{u}$ by divergence-free polynomials. The first step of our analysis is to define a divergence-free function which is polynomial with respect to one variable and which is an optimal approximation of $\mathbf{u}$.

Let $r$ be a real number with $r \geq 1$. Given a function $\mathbf{u}=\left(u_{1}, u_{2}, u_{3}\right)$ in $\left(\mathbf{H}_{\omega}^{r}(\Omega)\right)^{3}$, not necessarily divergence-free, we define $\varphi_{1}$ and $\varphi_{2}$ by

$$
\varphi_{1}(\mathbf{x})=-\int_{-1}^{z} u_{2}(x, y, t) d t \quad \text { and } \quad \varphi_{2}(\mathbf{x})=\int_{-1}^{z} u_{1}(x, y, t) d t .
$$

We define the operator $\wp_{r, N}^{z}$ as follows:

$$
\forall \mathbf{u} \in\left(\mathbf{H}_{\omega}^{r}(\Omega)\right)^{3}, \quad\left(\begin{array}{l}
\left(\wp_{r, N}^{z} \mathbf{u}\right)_{1}=\frac{\partial}{\partial z} \Pi_{r, N}^{z} \varphi_{2}, \\
\left(\wp_{r, N}^{z} \mathbf{u}\right)_{2}=-\frac{\partial}{\partial z} \Pi_{r, N}^{z} \varphi_{1}, \\
\left(\wp_{r, N}^{z} \mathbf{u}\right)_{3}=\Pi_{r, N}^{z} u_{3} .
\end{array}\right.
$$

The operators $\wp_{r, N}^{x}$ and $\wp_{r, N}^{y}$ are defined analogously.

Remark 2.1. Assume that $\mathbf{u}$ is a divergence-free function. Then we get

$$
u_{1}=\frac{\partial \varphi_{2}}{\partial z}, \quad u_{2}=-\frac{\partial \varphi_{1}}{\partial z} \quad \text { and } \quad u_{3}=\frac{\partial \varphi_{1}}{\partial y}-\frac{\partial \varphi_{2}}{\partial x}
$$

and using (2.9), we deduce that $\wp_{r, N}^{z} \mathbf{u}$ is also divergence-free.

Remark 2.2. Let $r$ be a real number such that $r \geq 1$ and $\mathbf{u}$ be an element of $\left(\mathbf{H}_{\omega}^{r}(\Omega)\right)^{3} \cap\left(\mathbf{H}_{\omega, 0}^{1}(\Omega)\right)^{3}$. Using (2.8) and (2.10), we clearly have

$$
\begin{array}{lll}
\left(\wp_{r, N}^{z} \mathbf{u}\right)_{1}(-1, y, z)=\left(\wp_{r, N}^{z} \mathbf{u}\right)_{1}(1, y, z)=0 & \text { a.e. }(y, z) \text { in } \Lambda^{2}, \\
\left(\wp_{r, N}^{z} \mathbf{u}\right)_{1}(x,-1, z)=\left(\wp_{r, N}^{z} \mathbf{u}\right)_{1}(x, 1, z)=0 & \text { a.e. }(x, z) \text { in } \Lambda^{2},
\end{array}
$$

and the same properties hold for $\left(\wp_{r, N}^{z} \mathbf{u}\right)_{2}$ and $\left(\wp_{r, N}^{z} \mathbf{u}\right)_{3}$. Since $r$ is such that $r \geq 1$, we have also

$$
\left(\wp_{r, N}^{z} \mathbf{u}\right)_{3}(x, y,-1)=\left(\wp_{r, N}^{z} \mathbf{u}\right)_{3}(x, y, 1)=0 \quad \text { a.e. }(x, y) \text { in } \Lambda^{2} .
$$

Assume now that $r$ is such that $r>1+\sigma_{0}$. Using (2.3), (2.8) and (2.10), we obtain

$$
\left(\wp_{r, N}^{z} \mathbf{u}\right)_{1}(x, y,-1)=\left(\wp_{r, N}^{z} \mathbf{u}\right)_{1}(x, y, 1)=0 \text { a.e. }(x, y) \text { in } \Lambda^{2} \text {, }
$$

and the same property holds for $\left(\wp_{r, N}^{z} \mathbf{u}\right)_{2}$.

We now give an estimate for the quantity $\mathbf{u}-\wp_{r, N}^{z} \mathbf{u}$. We will find that for any real number $r \geq 2$ and for any function $\mathbf{u}$ in $\left(\mathbf{H}_{\omega}^{r}(\Omega)\right)^{3} \cap\left(\mathbf{H}_{\omega, 0}^{1}(\Omega)\right)^{3}$, the function $\wp_{r, N}^{z} \mathbf{u}$ also belongs to $\left(\mathbf{H}_{\omega, 0}^{1}(\Omega)\right)^{3}$. 
THEOREM 2.2. Let $r$ be a real number with $r \geq 1$ and $r \notin \mathbb{N}+\sigma_{0}$, and $N$ be an integer with $N \geq 2\left[r-\sigma_{0}\right]+1$. For any integer $\nu$ with $0 \leq \nu \leq r-1$, and for any real number $\sigma$ with $\sigma \geq r+\nu$, there exists a positive constant $C$ independent of $N$ such that

$$
\forall \mathbf{u} \in\left(\mathbf{H}_{\omega}^{\sigma}(\Omega)\right)^{3}, \quad\left\|\mathbf{u}-\wp_{r, N}^{z} \mathbf{u}\right\|_{\nu, \omega} \leq C N^{\nu-\sigma}\|\mathbf{u}\|_{\sigma, \omega} .
$$

Moreover, if $r$ is a real number such that $r \geq 2$, then the estimate (2.13) is still valid for any real numbers $\nu$ and $\sigma$ such that $0 \leq \nu,[\nu] \leq r-2$ and $\sigma \geq r+1+[\nu]$.

Proof. Let $r$ be a real number such that $r \geq 1$ and $r \notin \mathbb{N}+\sigma_{0}, \nu$ be an integer with $0 \leq \nu \leq r-1, \sigma$ be a real number such that $\sigma \geq \nu+r$ and $\mathbf{u}$ be an element of $\left(\mathbf{H}_{\omega}^{r}(\Omega)\right)^{3}$.

(1) We estimate the first component of $\mathbf{u}-\wp_{r, N}^{z} \mathbf{u}$. Using (2.9) and (2.10), we obtain

$$
\left\|u_{1}-\left(\wp_{r, N}^{z} \mathbf{u}\right)_{1}\right\|_{\nu, \omega}^{2}=\sum_{\substack{\mathbf{k} \in \mathbf{N}^{3} \\|\mathbf{k}|_{1} \leq \nu}}\left\|\frac{\partial^{k_{3}+1}}{\partial z^{k_{3}+1}}\left(\frac{\partial^{k_{1}+k_{2}} \varphi_{2}}{\partial x^{k_{1}} \partial y^{k_{2}}}-\Pi_{r, N}^{z} \frac{\partial^{k_{1}+k_{2}} \varphi_{2}}{\partial x^{k_{1}} \partial y^{k_{2}}}\right)\right\|_{0, \omega}^{2} .
$$

Since we have $k_{3}+1 \leq \nu+1 \leq r$ and $\sigma-\nu+k_{3}+1 \geq \sigma-\nu+1 \geq r+1$, using Corollary 2.1, we obtain

$$
\begin{aligned}
\left\|u_{1}-\left(\wp_{r, N}^{z} \mathbf{u}\right)_{1}\right\|_{\nu, \omega}^{2} \leq & C \sum_{\substack{\mathbf{k} \in \mathbf{N}^{3} \\
|\mathbf{k}|_{1} \leq \nu}} N^{2\left(k_{3}+1\right)-2\left(\sigma-\nu+k_{3}+1\right)} \\
& \times \sum_{0 \leq j \leq \sigma-\nu+k_{3}+1}\left\|\frac{\partial^{j}}{\partial z^{j}}\left(\frac{\partial^{k_{1}+k_{2}} \varphi_{2}}{\partial x^{k_{1}} \partial y^{k_{2}}}\right)\right\|_{0, \omega}^{2} \\
\leq & C N^{2(\nu-\sigma)}\left\{\left\|\varphi_{2}\right\|_{\sigma, \omega}^{2}+\left\|\frac{\partial \varphi_{2}}{\partial z}\right\|_{\sigma, \omega}^{2}\right\} .
\end{aligned}
$$

We note that for any $\mathbf{k}$ in $\mathbb{N}^{2}, \partial^{k_{1}+k_{2}} \varphi_{2} / \partial x^{k_{1}} \partial y^{k_{2}}$ vanishes for $z=-1$; hence, applying the Poincaré inequality (see [9, Lemma 1.1] for the case of the Chebyshev weight), we obtain

$$
\left\|u_{1}-\left(\wp_{r, N}^{z} \mathbf{u}\right)_{1}\right\|_{\nu, \omega} \leq C N^{\nu-\sigma}\left\|\frac{\partial \varphi_{2}}{\partial z}\right\|_{\sigma, \omega}=C N^{\nu-\sigma}\left\|u_{1}\right\|_{\sigma, \omega} .
$$

(2) The second component of $\mathbf{u}-\wp_{r, N}^{z} \mathbf{u}$ can be estimated in the same way.

(3) We now estimate the third component of $\mathbf{u}-\wp_{r, N}^{z} \mathbf{u}$. We have

$$
\left\|u_{3}-\left(\wp_{r, N}^{z} \mathbf{u}\right)_{3}\right\|_{\nu, \omega}^{2}=\sum_{\substack{\mathbf{k} \in N^{3} \\|\mathbf{k}|_{1} \leq \nu}}\left\|\frac{\partial^{k_{3}}}{\partial z^{k_{3}}}\left(\frac{\partial^{k_{1}+k_{2}} u_{3}}{\partial x^{k_{1}} \partial y^{k_{2}}}-\Pi_{r, N}^{z} \frac{\partial^{k_{1}+k_{2}} u_{3}}{\partial x^{k_{1}} \partial y^{k_{2}}}\right)\right\|_{0, \omega}^{2} .
$$

Since we have $k_{3} \leq \nu \leq r-1$ and $\sigma-\nu+k_{3} \geq \sigma-\nu \geq r$, using Corollary 2.1, we obtain

$$
\begin{aligned}
\left\|u_{3}-\left(\wp_{r, N}^{z} \mathbf{u}\right)_{3}\right\|_{\nu, \omega}^{2} \leq & C \sum_{\substack{\mathbf{k} \in N^{3} \\
|\mathbf{k}|_{1} \leq \nu}} N^{2 k_{3}-2\left(\sigma-\nu+k_{3}\right)} \\
& \times \sum_{\substack{0 \leq j \leq \sigma-\nu+k_{3}\\
}}\left\|\frac{\partial^{j}}{\partial z^{j}}\left(\frac{\partial^{k_{1}+k_{2}} \varphi_{2}}{\partial x^{k_{1}} \partial y^{k_{2}}}\right)\right\|_{0, \omega}^{2} \\
& C N^{2(\nu-\sigma)}\left\|u_{3}\right\|_{\sigma, \omega}^{2} .
\end{aligned}
$$


Thus the estimate (2.13) is established when $\nu$ is an integer.

Finally, if $r$ and $\nu$ and $\sigma$ are three real numbers such that $r \geq 2,0 \leq[\nu]$, $([\nu]+1) \leq r-1$ and $r \leq \sigma-([\nu]+1)$, we obtain (2.13) by interpolation between $\left(\mathbf{H}_{\omega}^{[\nu]}(\Omega)\right)^{3}$ and $\left(\mathbf{H}_{\omega}^{[\nu]+1}(\Omega)\right)^{3}$. This completes the proof.

Remark 2.3. Let $r$ be a real number such that $r \geq 2$ and $\mathbf{u}$ be an element of the space $\left(\mathbf{H}_{\omega}^{r+1}(\Omega)\right)^{3} \cap\left(\mathbf{H}_{\omega, 0}^{1}(\Omega)\right)^{3}$. Using Remark 2.2, and applying (2.13) with $\nu=1$ and $\sigma=r+1$, we obtain that $\wp_{r, N}^{z} \mathbf{u}$ is an element of $\left(\mathbf{H}_{\omega, 0}^{1}(\Omega)\right)^{3}$.

Remark 2.4. Let $r$ be a real number such that $r \geq 1$. For any function $\mathbf{u}$ in $\left(\mathbf{H}_{\omega}^{r}(\Omega)\right)^{3}$, we have

$$
\operatorname{div} \wp_{r, N}^{z} \mathbf{u}(\mathbf{x})=\frac{\partial}{\partial z}\left(\Pi_{r, N}^{z}\left(\int_{-1}^{z} \operatorname{div} \mathbf{u}(x, y, t) d t\right)\right) \quad \text { a.e. } \mathbf{x} \text { in } \Omega
$$

Following the proof of Theorem 2.2, we obtain the following result:

If $r$ is a real number such that $r \geq 1$ and $r \notin \mathbf{N}+\sigma_{0}$, then for any integer $\nu$ such that $\nu \leq r-1$ and for any real number $\sigma$ such that $\sigma \geq r+\nu$, there exists a positive constant $C$ independent of $N$ such that

$$
\forall \mathbf{u} \in\left(\mathbf{H}_{\omega}^{\sigma}(\Omega)\right)^{3}, \quad\left\|\operatorname{div} \mathbf{u}-\operatorname{div} \wp_{r, N}^{z} \mathbf{u}\right\|_{\nu, \omega} \leq C N^{\nu+1-\sigma}\|\operatorname{div} \mathbf{u}\|_{\sigma-1, \omega}
$$

Moreover, if $r$ is such that $r \geq 2$, then the preceding estimate holds for any real numbers $\nu$ and $\sigma$ such that $0 \leq \nu,[\nu] \leq r-2$ and $\sigma \geq r+[\nu]+1$.

Let $r$ be a real number with $r \geq 1$. For any divergence-free function $\mathbf{u}$ in $\left(\mathbf{H}_{\omega}^{r}(\Omega)\right)^{3} \cap\left(\mathbf{H}_{\omega, 0}^{1}(\Omega)\right)^{3}$, we denote by $\wp_{r, N}^{\text {div }} \mathbf{u}$ the orthogonal projection of $\mathbf{u}$ for the inner product of $\left(\mathbf{H}_{\omega}^{r}(\Omega)\right)^{3}$ onto the space of divergence-free polynomials of degree $\leq N$ which vanish on the boundary of $\Omega$.

We now state the main theorem of this section.

THEOREM 2.3. Let $r$ and $N$ be two integers with $r \geq 1$ and $N \geq 0$. For any real number $\sigma$ with $\sigma \geq 2 r+1$, there exists a positive constant $C$ independent of $N$ such that

(2.14) $\forall \mathbf{u} \in\left(\mathbf{H}_{\omega}^{\sigma}(\Omega)\right)^{3} \cap\left(\mathbf{H}_{\omega, 0}^{1}(\Omega)\right)^{3} ; \operatorname{div} \mathbf{u}=0, \quad\left\|\mathbf{u}-\wp_{r, N}^{\operatorname{div}} \mathbf{u}\right\|_{r, \omega} \leq C N^{r-\sigma}\|\mathbf{u}\|_{\sigma, \omega}$.

Moreover, if $r$ is a real number such that $r \geq 1$ and $r \notin \mathbf{N}+\sigma_{0}$, the estimate (2.14) is still valid for any real number $\sigma$ such that $\sigma \geq r+[r]+3$.

Proof. Let $r$ be an integer with $r \geq 1$. In order to prove (2.14), we may assume that $N$ is such that $N \geq 2\left[r-\sigma_{0}\right]+3$. We denote by $\wp_{r, N}^{z \text {, div }}$ the orthogonal projection operator for the inner product of $\left(\mathbf{H}_{\omega}^{r}(\Omega)\right)^{3}$ onto the subspace of $\left(\mathbf{H}_{\omega, 0}^{1}(\Omega)\right)^{3}$ of divergence-free functions which are polynomial of degree $\leq N$ in the $z$ variable. We define $\wp_{r, N}^{x, \text { div }}$ and $\wp_{r, N}^{y, d i v}$ in the same way.

Let $\sigma$ be a real number with $\sigma \geq 2 r+1$ and $\mathbf{u}$ be a divergence-free element of $\left(\mathbf{H}_{\omega}^{\sigma}(\Omega)\right)^{3} \cap\left(\mathbf{H}_{\omega, 0}^{\mathbf{1}}(\Omega)\right)^{3}$.

We have $\sigma \geq 2 r+1 \geq r+2$. Since $\mathbf{u}$ belongs to $\left(\mathbf{H}_{\omega}^{\sigma}(\Omega)\right)^{3} \cap\left(\mathbf{H}_{\omega, 0}^{1}(\Omega)\right)^{3}$, we deduce from Remarks 2.1 and 2.3 that $\wp_{r+1, N}^{z} \mathrm{u}$ is a divergence-free function which belongs to the subspace of $\left(\mathbf{H}_{\omega, 0}^{1}(\Omega)\right)^{3}$ of divergence-free functions which are polynomial of degree $\leq N$ in the $z$ variable. We deduce from the definition of $\wp_{r, N}^{z, \text { div }}$ that

$$
\left\|\mathbf{u}-\wp_{r, N}^{\boldsymbol{z}, \operatorname{div}} \mathbf{u}\right\|_{r, \omega} \leq\left\|\mathbf{u}-\wp_{r+1, N}^{z} \mathbf{u}\right\|_{r, \omega}
$$


hence, using Theorem 2.2, we obtain

$$
\left\|\mathbf{u}-\wp_{r, N}^{z, \operatorname{div}} \mathbf{u}\right\|_{r, \omega} \leq C N^{r-\sigma}\|\mathbf{u}\|_{\sigma, \omega}
$$

Moreover, from the definition of $\wp_{r, N}^{z, \text { div }}$ we also have

$$
\forall \mathbf{v} \in\left(\mathbf{H}_{\omega}^{r}(\Omega)\right)^{3} \cap\left(\mathbf{H}_{\omega, 0}^{1}(\Omega)\right)^{3} ; \operatorname{div} \mathbf{v}=0, \quad\left\|\mathbf{v}-\wp_{r, N}^{z, \operatorname{div}} \mathbf{v}\right\|_{r, \omega} \leq\|\mathbf{v}\|_{r, \omega} .
$$

Naturally, the estimates (2.15) and (2.16) hold also for $\wp_{r, N}^{x, \operatorname{div}}$ and $\wp_{r, N}^{y, \operatorname{div}}$.

The divergence-free function $\wp_{r, N}^{x, \text { div }} \wp_{r, N}^{y, \text { div }} \wp_{r, N}^{z, \text { div }} \mathbf{u}$ is a polynomial of degree $\leq N$ which vanishes on the boundary of $\Omega$. Hence we deduce

$$
\left\|\mathbf{u}-\wp_{r, N}^{\operatorname{div}} \mathbf{u}\right\|_{r, \omega} \leq\left\|\mathbf{u}-\wp_{r, N}^{x, \operatorname{div}} \wp_{r, N}^{y, \operatorname{div}} \wp_{r, N}^{z, \operatorname{div}} \mathbf{u}\right\|_{r, \omega} .
$$

To estimate the right-hand side of $(2.17)$, we note that

$$
\begin{aligned}
\mathbf{u}-\wp_{r, N}^{x, \operatorname{div}} \wp_{r, N}^{y, \operatorname{div}} \wp_{r, N}^{z, \operatorname{div}} \mathbf{u}= & \left(\mathbf{u}-\wp_{r, N}^{x, \operatorname{div}} \mathbf{u}\right)+\left(\mathbf{u}-\wp_{r, N}^{y, \operatorname{div}} \wp_{r, N}^{z, \operatorname{div}} \mathbf{u}\right) \\
& -\left(\mathbf{I}-\wp_{r, N}^{x, \operatorname{div}}\right)\left(\mathbf{u}-\wp_{r, N}^{y, \operatorname{div}} \wp_{r, N}^{z, \operatorname{div}} \mathbf{u}\right)
\end{aligned}
$$

Therefore, by (2.16), we deduce

$$
\begin{aligned}
\| \mathbf{u}- & \wp_{r, N}^{x, \operatorname{div}} \wp_{r, N}^{y, \operatorname{div}} \wp_{r, N}^{z, \operatorname{div}} \mathbf{u} \|_{r, \omega} \\
& \leq\left\|\mathbf{u}-\wp_{r, N}^{x, \operatorname{div}} \mathbf{u}\right\|_{r, \omega}+2\left\|\mathbf{u}-\wp_{r, N}^{y, \operatorname{div}} \wp_{r, N}^{z, \operatorname{div}} \mathbf{u}\right\|_{r, \omega}
\end{aligned}
$$

and proceeding in the same way, we finally obtain

$$
\begin{aligned}
\| \mathbf{u}- & \wp_{r, N}^{x, \operatorname{div}} \wp_{r, N}^{y, \operatorname{div}} \wp_{r, N}^{z, \operatorname{div}} \mathbf{u} \|_{r, \omega} \\
& \leq\left\|\mathbf{u}-\wp_{r, N}^{x, \operatorname{div}} \mathbf{u}\right\|_{r, \omega}+2\left\|\mathbf{u}-\wp_{r, N}^{y, \operatorname{div}} \mathbf{u}\right\|_{r, \omega}+3\left\|\mathbf{u}-\wp_{r, N}^{z, \operatorname{div}} \mathbf{u}\right\|_{r, \omega} .
\end{aligned}
$$

From (2.15), (2.17) and (2.18) we obtain (2.14).

If $r$ is a real number such that $r \notin \mathbb{N}+\sigma_{0}$, the same proof, where the operator $\wp_{r+1, N}^{z}$ is replaced by $\wp_{r+2, N}^{z}$, gives the result for any real number $\sigma$ such that $\sigma \geq r+[r]+3$.

Remark 2.5. For any real number $s \geq 1$, set

$$
\mathbf{H}_{\omega}^{\circ s, \operatorname{div}}(\Omega)=\left\{\mathbf{u} \in\left(\mathbf{H}_{\omega}^{s}(\Omega)\right)^{3} \cap\left(\mathbf{H}_{\omega, 0}^{1}(\Omega)\right)^{3} ; \operatorname{div} \mathbf{u}=0\right\} .
$$

Let $r$ be a real number with $r \geq 1$. Theorem 2.3 shows that for any real number $\sigma$ such that $\sigma \geq r+[r]+3$, the operator $\mathbf{I}-\wp_{r, N}^{\mathrm{div}}$ is continuous from $\mathbf{H}_{\omega}^{\circ \sigma}{ }_{\omega}^{\text {, div }}(\Omega)$ into $\left(\mathbf{H}_{\omega}^{r}(\Omega)\right)^{3}$ with a norm $\leq C N^{r-\sigma}$. On the other hand, it is clear that $\mathbf{I}-\wp_{r, N}^{\text {div }}$ is continuous from $\mathbf{H}_{\omega}^{\circ}{ }_{\omega}$, div $(\Omega)$ into $\left(\mathbf{H}_{\omega}^{r}(\Omega)\right)^{3}$ with a norm equal to 1 . Then we could obtain the estimate (2.14) for any real number $\sigma \geq r$ by using an interpolation argument, if the following property were satisfied:

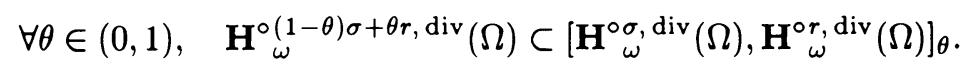

Unfortunately, even if $\omega$ is the Legendre weight function, we are not able to prove the property (2.19). Meanwhile, when the function $\mathbf{u}$ is assumed to be periodic in some directions, we can prove the estimate (2.14) for any real number $\sigma \geq r$ by using an interpolation result similar to (2.19) (see Section 3 and the Appendix).

Remark 2.6. Let $r$ be a real number such that $r \notin \mathbb{N}+\sigma_{0}$ and $m$ be an integer such that $0 \leq m \leq r$. For any divergence-free function $\mathbf{u}$ in $\left(\mathbf{H}_{\omega}^{r}(\Omega)\right)^{3} \cap\left(\mathbf{H}_{\omega, 0}^{m}(\Omega)\right)^{3}$, we denote by $\wp_{r, N}^{m, \text { div }} \mathbf{u}$ the orthogonal projection of $\mathbf{u}$ for the inner product of 
$\left(\mathbf{H}_{\omega}^{r}(\Omega)\right)^{3}$ onto the space of divergence-free polynomials of degree $\leq N$ which belong to $\left(\mathbf{H}_{\omega, 0}^{m}(\Omega)\right)^{3}$. Using the same technique as above, we can prove the following result: for any real number $\sigma$ such that $\sigma \geq 2 r+1$ (resp. $\sigma \geq r+[r]+3$ ) when $r$ is an integer (resp. a real number), there exists a positive constant $C$ independent of $N$ such that

$$
\forall \mathbf{u} \in\left(\mathbf{H}_{\omega}^{\sigma}(\Omega)\right)^{3} \cap\left(\mathbf{H}_{\omega, 0}^{m}(\Omega)\right)^{3} ; \operatorname{div} \mathbf{u}=0, \quad\left\|\mathbf{u}-\wp_{r, N}^{m, \operatorname{div}} \mathbf{u}\right\|_{r, \omega} \leq C N^{r-\sigma}\|\mathbf{u}\|_{\sigma, \omega} .
$$

If $\mathbf{u}$ is a function of two variables, the estimate (2.14) can be obtained for any real number $\sigma \geq r$ by using a simpler technique. Precisely, the following result has been proved in [25] and [5]:

THEOREM 2.4. Let $r$ be a real number such that $r \geq 1$ and $r \notin \mathbb{N}+\sigma_{0}$. For any real number $\sigma$ with $\sigma \geq r$, there exists a positive constant $C$ independent of $N$ such that

$$
\begin{aligned}
\forall \mathbf{u} \in\left(\mathbf{H}_{\omega}^{r}\left(\Lambda^{2}\right)\right)^{2} \cap\left(\mathbf{H}_{\omega, 0}^{1}\left(\Lambda^{2}\right)\right)^{2} ; \operatorname{div} \mathbf{u} & =0 \\
& \left\|\mathbf{u}-\wp_{r, N}^{\operatorname{div}} \mathbf{u}\right\|_{r, \omega} \leq C N^{r-\sigma}\|\mathbf{u}\|_{\sigma, \omega}
\end{aligned}
$$

2.3. A Different Approach in the Case of the Legendre Weight. In the Legendre case (i.e., when $\omega=1$ ), we now consider the problem of the best approximation of both a function and its divergence.

For any function $\mathbf{u}$ in $\left(\mathbf{H}_{0}^{1}(\Omega)\right)^{3}$ (not necessarily divergence-free), we shall define a polynomial $\Pi_{N} \mathbf{u}$ such that the divergence of $\Pi_{N} \mathbf{u}$ is related to the $\mathbf{H}^{1}$-projection of the divergence of $\mathbf{u}$. A relation of this kind is useful in the applications. For any integer $d$, the same type of result can be stated in the same way for a function $\mathbf{u}$ of $\left(\mathbf{H}_{0}^{1}\left(\Lambda^{d}\right)\right)^{d}$ (see Remark 2.9).

We denote by $\left(L_{n}\right)_{n \in \mathbb{N}}$ the sequence of Legendre polynomials defined by the recurrence relation

$$
\forall x \in \Lambda, \quad\left(\begin{array}{l}
L_{0}(x)=1, \quad L_{1}(x)=x \\
\forall n \geq 2, \quad n L_{n}(x)=(2 n-1) x L_{n-1}(x)-(n-1) L_{n-2}(x) .
\end{array}\right.
$$

We recall the following relations:

$$
\left\{\begin{array}{l}
\forall n \geq 0, \quad L_{n}(1)=1, \quad L_{n}(-1)=(-1)^{n}, \\
\forall n \geq 1, \quad \frac{L_{n+1}^{\prime}-L_{n-1}^{\prime}}{2 n+1}=L_{n}, \\
\forall v \in \mathbf{L}^{2}(\Lambda), \quad v=\sum_{\mathrm{k}=0}^{\infty} \frac{2 k+1}{2}\left(v, L_{k}\right) L_{k} .
\end{array}\right.
$$

We denote by $\Pi_{0, N}$ the orthogonal projection operator on $\mathbf{P}_{N}(\Lambda)$ with respect to the $\mathbf{L}^{2}(\Lambda)$-inner product. We recall that the operator $\Pi_{1, N}^{\circ 1}$ introduced in Theorem 2.1 is the projection operator on $\mathbf{P}_{N}(\Lambda) \cap \mathbf{H}_{0}^{1}(\Lambda)$ with respect to the $\mathbf{H}_{0}^{1}(\Lambda)$-inner product, i.e., it satisfies

$$
\forall \varphi \in \mathbf{H}_{0}^{1}(\Lambda), \quad\left(\begin{array}{l}
\Pi_{1, N}^{\circ 1} \varphi \in \mathbf{P}_{N}(\Lambda) \cap \mathbf{H}_{0}^{1}(\Lambda), \\
\forall \psi \in \mathbf{P}_{N}(\Lambda) \cap \mathbf{H}_{0}^{1}(\Lambda), \quad\left(\left(\Pi_{1, N}^{\circ 1} \varphi\right)^{\prime}, \psi^{\prime}\right)=\left(\varphi^{\prime}, \psi^{\prime}\right) .
\end{array}\right.
$$

We first state a relation between $\Pi_{0, N-2}$ and $\Pi_{1, N}^{\circ 1}$.

LEMMA 2.1. For any function $\varphi$ in $\mathbf{H}_{0}^{1}(\Lambda)$, the following formula holds:

$$
\Pi_{1, N}^{\circ 1} \varphi=\Pi_{0, N-2} \varphi-\frac{1}{2}\left(\varphi, L_{N-2}^{\prime}\right) L_{N-1}-\frac{1}{2}\left(\varphi, L_{N-1}^{\prime}\right) L_{N}
$$


Proof. Let $Q$ be an element of $\mathbf{P}_{N-2}(\Lambda)$ and let $R$ be the element of $\mathbf{P}_{N}(\Lambda) \cap$ $\mathbf{H}_{0}^{1}(\Lambda)$ such that $R^{\prime \prime}=Q$. Using $(2.22)$, for any function $\varphi$ in $\mathbf{H}_{0}^{1}(\Lambda)$ we have

$$
\left(\Pi_{1, N}^{\circ 1} \varphi, Q\right)=-\left(\left(\Pi_{1, N}^{\circ 1} \varphi\right)^{\prime}, R^{\prime}\right)=-\left(\varphi^{\prime}, R^{\prime}\right)=(\varphi, Q) \text {. }
$$

Hence we deduce

$$
\begin{aligned}
\Pi_{1, N}^{\circ 1} \varphi= & \Pi_{0, N-2} \varphi-\frac{2 N-1}{2}\left(\Pi_{1, N}^{\circ 1} \varphi, L_{N-1}\right) L_{N-1} \\
& -\frac{2 N+1}{2}\left(\Pi_{1, N}^{\circ 1} \varphi, L_{N}\right) L_{N} .
\end{aligned}
$$

We now compute the last two terms in the right-hand side of (2.24). Let $j$ be an element of $\{0,1\}$. Using $(2.21)$, we get

$$
\begin{aligned}
\left(\Pi_{1, N}^{\circ 1} \varphi, L_{N-j}\right) & =\left(\Pi_{1, N}^{\circ 1} \varphi, \frac{L_{N+1-j}^{\prime}-L_{N-1-j}^{\prime}}{2 N-2 j+1}\right) \\
& =-\left(\left(\Pi_{1, N}^{\circ} \varphi\right)^{\prime}, \frac{L_{N+1-j}-L_{N-1-j}}{2 N-2 j+1}\right) .
\end{aligned}
$$

We note that $N+1-j \geq N$ and that $\left(\Pi^{\circ 1}{ }_{1, N} \varphi\right)^{\prime}$ is of degree at most $N-1$. Hence we have

$$
\left(\Pi_{1, N}^{\circ 1} \varphi, L_{N-j}\right)=\left(\left(\Pi_{1, N}^{\circ 1} \varphi\right)^{\prime}, \frac{L_{N-1-j}}{2 N-2 j+1}\right) .
$$

Using again (2.21), we obtain

$$
\left(\Pi_{1, N}^{\circ 1} \varphi, L_{N-j}\right)=\frac{1}{2 N-2 j+1}\left(\left(\Pi_{1, N}^{\circ 1} \varphi\right)^{\prime}, \frac{L_{N-j}^{\prime}-L_{N-2-j}^{\prime}}{2 N-2 j-1}\right) .
$$

Since the polynomial $L_{N-j}-L_{N-2-j}$ belongs to $\mathbf{P}_{N}(\Lambda) \cap \mathbf{H}_{0}^{1}(\Lambda)$, using (2.22) we deduce

$$
\begin{aligned}
\left(\Pi_{1, N}^{\circ 1} \varphi, L_{N-j}\right) & =\frac{1}{2 N-2 j+1}\left(\varphi^{\prime}, \frac{L_{N-j}^{\prime}-L_{N-2-j}^{\prime}}{2 N-2 j-1}\right) \\
& =\left(\varphi^{\prime}, \frac{L_{N-1-j}}{2 N-2 j+1}\right)=-\left(\varphi, \frac{L_{N-1-j}^{\prime}}{2 N-2 j+1}\right),
\end{aligned}
$$

and applying (2.24), this completes the proof of (2.23).

We now define an operator $\Pi_{N}^{*}$ by

$$
\forall \varphi \in \mathbf{H}^{2}(\Lambda), \forall x \in \Lambda, \quad\left(\Pi_{N}^{*} \varphi\right)(x)=\int_{-1}^{x}\left(\Pi_{1, N-1} \varphi^{\prime}\right)(t) d t
$$

LEMMA 2.2. For any function $\varphi$ in $\mathbf{H}^{2}(\Lambda) \cap \mathbf{H}_{0}^{1}(\Lambda), \Pi_{N}^{*} \varphi$ is an element of $\mathbf{P}_{N}(\Lambda) \cap \mathbf{H}_{0}^{1}(\Lambda)$ such that $\left(\Pi_{N}^{*} \varphi\right)^{\prime}( \pm 1)=\varphi^{\prime}( \pm 1)$. Moreover, for any real numbers $\nu$ and $\sigma$ with $0 \leq \nu \leq 2 \leq \sigma$, there exists a constant $C$ independent of $N$ such that

$$
\forall \varphi \in \mathbf{H}^{\sigma}(\Lambda) \cap \mathbf{H}_{0}^{1}(\Lambda), \quad\left\|\varphi-\Pi_{N}^{*} \varphi\right\|_{\nu} \leq C N^{\nu-\sigma}\|\varphi\|_{\sigma} .
$$

Proof. Let $\varphi$ be an element of $\mathbf{H}^{2}(\Lambda) \cap \mathbf{H}_{0}^{1}(\Lambda)$.

(1) Let $q$ be an element of $\mathbf{P}_{1}(\Lambda)$ such that $q( \pm 1)=\varphi^{\prime}( \pm 1)$. From (2.6) we have

$$
\Pi_{1, N-1}\left(\varphi^{\prime}\right)=q+\Pi_{1, N-1}^{\circ 1}\left(\varphi^{\prime}-q\right) \text {. }
$$

From Lemma 2.1 we deduce

$$
\int_{-1}^{1} \Pi^{\circ 1}{ }_{1, N-1}\left(\varphi^{\prime}-q\right)(t) d t=\int_{-1}^{1}\left(\varphi^{\prime}-q\right)(t) d t
$$


hence

$$
\int_{-1}^{1} \Pi_{1, N-1}\left(\varphi^{\prime}\right)(t) d t=\int_{-1}^{1} \varphi^{\prime}(t) d t=0
$$

and $\Pi_{N}^{*} \varphi$ belongs to $\mathbf{P}_{N}(\Lambda) \cap \mathbf{H}_{0}^{1}(\Lambda)$. The fact that $\left(\Pi_{N}^{*} \varphi\right)^{\prime}( \pm 1)=\varphi^{\prime}( \pm 1)$ follows from (2.6) and (2.25).

(2) We now prove (2.26). We first observe that

$$
\left\|\varphi-\Pi_{N}^{*} \varphi\right\|_{1} \leq C\left\|\varphi^{\prime}-\Pi_{1, N-1} \varphi^{\prime}\right\|_{0}
$$

and

$$
\left\|\varphi-\dot{\Pi}_{N}^{*} \varphi\right\|_{2} \leq C\left\|\varphi^{\prime}-\Pi_{1, N-1} \varphi^{\prime}\right\|_{1}
$$

hence, from (2.4), we deduce (2.26) for $\nu=1$ and $\nu=2$. In order to prove (2.26) for $\nu=0$, we use a duality argument. For any $\psi$ in $\mathrm{L}^{2}(\Lambda)$, we denote by $A \psi$ the element of $\mathbf{H}^{2}(\Lambda) \cap \mathbf{H}_{0}^{1}(\Lambda)$ such that $-(A \psi)^{\prime \prime}=\psi$. We have

$$
\left\|\varphi-\Pi_{N}^{*} \varphi\right\|_{0}=\sup _{\psi \in \mathbf{L}^{2}(\Lambda)} \frac{\left(\varphi-\Pi_{N}^{*} \varphi, \psi\right)}{\|\psi\|_{0}} .
$$

Since $\varphi-\Pi_{N}^{*} \varphi$ belongs to $\mathbf{H}_{0}^{1}(\Lambda)$, we deduce

$$
\left\|\varphi-\Pi_{N}^{*} \varphi\right\|_{0}=\sup _{\psi \in \mathbf{L}^{2}(\Lambda)} \frac{\left(\left(\varphi-\Pi_{N}^{*} \varphi\right)^{\prime},(A \psi)^{\prime}\right)}{\|\psi\|_{0}} .
$$

Now let $q$ be the element of $\mathbf{P}_{1}(\Lambda)$ such that $q( \pm 1)=\varphi^{\prime}( \pm 1)$. We have

$$
\left(\varphi-\Pi_{N}^{*} \varphi\right)^{\prime}=\varphi^{\prime}-\Pi_{1, N-1} \varphi^{\prime}=\left(\varphi^{\prime}-q\right)-\Pi_{1, N-1}^{\circ 1}\left(\varphi^{\prime}-q\right) .
$$

Formula (2.23) yields

$$
\begin{aligned}
\left(\varphi-\Pi_{N}^{*} \varphi\right)^{\prime}= & \left\{\left(\varphi^{\prime}-q\right)-\Pi_{0, N-3}\left(\varphi^{\prime}-q\right)\right\}+\frac{1}{2}\left(\varphi^{\prime}-q, L_{N-3}^{\prime}\right) L_{N-2} \\
& +\frac{1}{2}\left(\varphi^{\prime}-q, L_{N-2}^{\prime}\right) L_{N-1},
\end{aligned}
$$

and we conclude that for any $\psi$ in $\mathrm{L}^{2}(\Lambda)$ we have $\left(\left(\varphi-\Pi_{N}^{*} \varphi\right)^{\prime}, \Pi_{0, N-3}(A \psi)^{\prime}\right)=0$. Recalling (2.27), we obtain

$$
\begin{aligned}
\left\|\varphi-\Pi_{N}^{*} \varphi\right\|_{0} & =\sup _{\psi \in \mathbf{L}^{2}(\Lambda)} \frac{\left(\left(\varphi-\Pi_{N}^{*} \varphi\right)^{\prime},(A \psi)^{\prime}-\Pi_{0, N-3}(A \psi)^{\prime}\right)}{\|\psi\|_{0}} \\
& \leq\left\|\varphi-\Pi_{N}^{*} \varphi\right\|_{1} \sup _{\psi \in \mathbf{L}^{2}(\Lambda)} \frac{\left\|(A \psi)^{\prime}-\Pi_{0, N-3}(A \psi)^{\prime}\right\|_{0}}{\|\psi\|_{0}} .
\end{aligned}
$$

Since (2.26) has been established for $\nu=1$, we deduce

$$
\begin{aligned}
\left\|\varphi-\Pi_{N}^{*} \varphi\right\|_{0} & \leq C\left(N^{1-\sigma}\|\varphi\|_{\sigma}\right)\left(N^{-1} \sup _{\psi \in \mathbf{L}^{2}(\Lambda)} \frac{\|A \psi\|_{2}}{\|\psi\|_{0}}\right) \\
& \leq C N^{-\sigma}\|\varphi\|_{\sigma} .
\end{aligned}
$$

Thus, (2.26) is proved for $\nu=0$. Finally, we obtain (2.26) for $0 \leq \nu \leq 2$ by interpolation.

For any function $u$ in $\mathbf{H}^{2}(\Omega)$, we set

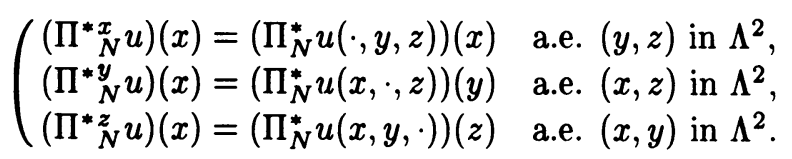


We note that $\Pi_{N}^{* z}$ commutes with differentiation with respect to the $x$ and $y$ variables, i.e.,

$$
\frac{\partial}{\partial x} \Pi_{N}^{* z} u=\Pi_{N}^{* z} \frac{\partial u}{\partial x} \text { and } \frac{\partial}{\partial y} \Pi_{N}^{* z} u=\Pi_{N}^{* z} \frac{\partial u}{\partial y},
$$

provided that the derivatives of $u$ belong to the domain of $\Pi^{* z}$.

For any $\mathbf{u}=\left(u_{1}, u_{2}, u_{3}\right)$ in $\left(\mathbf{H}^{4}(\Omega)\right)^{3}$, we define

$$
\left(\begin{array}{l}
\left(\Pi_{N} \mathbf{u}\right)_{1}=\Pi^{* x} \Pi_{1, N-1}^{y} \Pi_{1, N-1}^{z} u_{1}, \\
\left(\Pi_{N} \mathbf{u}\right)_{2}=\Pi_{1, N-1}^{x} \Pi^{*} y \Pi_{1, N-1}^{z} u_{2}, \\
\left(\Pi_{N} \mathbf{u}\right)_{3}=\Pi_{1, N-1}^{x} \Pi_{1, N-1}^{y} \Pi_{N}^{* z} u_{3} .
\end{array}\right.
$$

Remark 2.7. Set

$$
\begin{aligned}
& \vartheta_{1}=\left\{\mathbf{u} \in\left(\mathbf{L}_{\omega}^{2}(\Omega)\right)^{3} ; \forall(i, j, k) \in \mathbb{N}^{3}, i \leq 2, j \leq 1, k \leq 1, \frac{\partial^{i+j+k} u_{1}}{\partial x^{i} \partial y^{j} \partial z^{k}} \in \mathbf{L}_{\omega}^{2}(\Omega)\right\} \\
& \vartheta_{2}=\left\{\mathbf{u} \in\left(\mathbf{L}_{\omega}^{2}(\Omega)\right)^{3} ; \forall(i, j, k) \in \mathbb{N}^{3}, i \leq 1, j \leq 2, k \leq 1, \frac{\partial^{i+j+k} u_{2}}{\partial x^{i} \partial y^{j} \partial z^{k}} \in \mathbf{L}_{\omega}^{2}(\Omega)\right\}
\end{aligned}
$$

and

$$
\vartheta_{3}=\left\{\mathbf{u} \in\left(\mathbf{L}_{\omega}^{2}(\Omega)\right)^{3} ; \forall(i, j, k) \in \mathbb{N}^{3}, i \leq 1, j \leq 1, k \leq 2, \frac{\partial^{i+j+k} u_{3}}{\partial x^{i} \partial y^{j} \partial z^{k}} \in \mathbf{L}_{\omega}^{2}(\Omega)\right\} .
$$

It is easy to see that the domain of the operator $\Pi_{N}$ is the space $\vartheta_{1} \cap \vartheta_{2} \cap \vartheta_{3}$, which contains $\left(\mathbf{H}^{4}(\Omega)\right)^{3}$.

Remark 2.8. Let $\mathbf{u}$ be in $\left(\mathbf{H}^{4}(\Omega)\right)^{3} \cap\left(\mathbf{H}_{0}^{1}(\Omega)\right)^{3}$. From Corollary 2.1 and Lemma 2.2 we obtain that $\Pi_{N} \mathbf{u}$ is an element of $\left(\mathbf{P}_{N}(\Omega)\right)^{3} \cap\left(\mathbf{H}_{0}^{1}(\Omega)\right)^{3}$. Moreover, applying (2.25), it is clear that the divergence of $\Pi_{N} \mathbf{u}$ is given by

$$
\operatorname{div} \Pi_{N} \mathbf{u}=\Pi_{1, N-1}^{x} \Pi_{1, N-1}^{y} \Pi_{1, N-1}^{z} \operatorname{div} \mathbf{u} .
$$

Hence, if $\mathbf{u}$ is divergence-free, so is $\Pi_{N} \mathbf{u}$.

We are now in a position to estimate both $\mathbf{u}-\boldsymbol{\Pi}_{N} \mathbf{u}$ and $\operatorname{div} \mathbf{u}-\operatorname{div} \boldsymbol{\Pi}_{N} \mathbf{u}$.

THEOREM 2.5. For any real numbers $\nu$ and $\sigma$ such that $\sigma \geq 4$ and $0 \leq \nu \leq 1$, there exists a positive constant $C$ independent of $N$ such that

$$
\forall \mathbf{u} \in\left(\mathbf{H}^{\sigma}(\Omega)\right)^{3} \cap\left(\mathbf{H}_{0}^{1}(\Omega)\right)^{3}, \quad\left\|\mathbf{u}-\boldsymbol{\Pi}_{N} \mathbf{u}\right\|_{\nu} \leq C N^{\nu-\sigma}\|\mathbf{u}\|_{\sigma}
$$

and

(2.33) $\forall \mathbf{u} \in\left(\mathbf{H}^{\sigma}(\Omega)\right)^{3} \cap\left(\mathbf{H}_{0}^{1}(\Omega)\right)^{3}, \quad\left\|\operatorname{div} \mathbf{u}-\operatorname{div} \boldsymbol{\Pi}_{N} \mathbf{u}\right\|_{\nu} \leq C N^{\nu+1-\sigma}\|\operatorname{div} \mathbf{u}\|_{\sigma-1}$.

Remark 2.9. Let $d$ be an integer such that $d \geq 2$. For any function $\mathbf{u}$ in $\left(\mathbf{H}^{d+1}\left(\Lambda^{d}\right)\right)^{d}$, we define $\boldsymbol{\Pi}_{N} \mathbf{u}$ in the same way. We obtain the estimates (2.32) and (2.33) for any function $\mathbf{u}$ in $\left(\mathbf{H}^{\sigma}\left(\Lambda^{d}\right)\right)^{d} \cap\left(\mathbf{H}_{0}^{1}\left(\Lambda^{d}\right)\right)^{d}$ with $\sigma \geq d+1$.

Proof of Theorem 2.5. To simplify the notations, we prove the estimates (2.32) and (2.33) for a function $\mathbf{u}$ in $\left(\mathbf{H}^{\sigma}\left(\Lambda^{2}\right)\right)^{2} \cap\left(\mathbf{H}_{0}^{1}\left(\Lambda^{2}\right)\right)^{2}$ with $\sigma \geq 3$.

In order to prove (2.32), we prove the following inequality:

$$
\left\|u_{1}-\left(\boldsymbol{\Pi}_{N} \mathbf{u}\right)_{1}\right\|_{\nu} \leq C N^{\nu-\sigma}\left\|u_{1}\right\|_{\sigma}
$$

for $\sigma \geq 3$ and $0 \leq \nu \leq 1$; the estimate of $u_{2}-\left(\Pi_{N} \mathbf{u}\right)_{2}$ can be obtained in the same way. 
(1) We first prove (2.34) for $\nu=1$. The following inequality holds:

$$
\begin{aligned}
\left\|u_{1}-\left(\Pi_{N} \mathbf{u}\right)_{1}\right\|_{1} \leq & \left\|\left(I-\Pi_{N}^{* x}\right)\left(I-\Pi_{1, N-1}^{y}\right) u_{1}\right\|_{1}+\left\|\left(I-\Pi_{N}^{* x}\right) u_{1}\right\|_{1} \\
& +\left\|\left(I-\Pi_{1, N-1}^{y}\right) u_{1}\right\|_{1} .
\end{aligned}
$$

(1a) We treat the first term in the right-hand side of (2.35). Applying the Poincaré inequality, we observe that there exists a constant $C$ such that

$$
\begin{aligned}
\left\|\left(I-\Pi_{N}^{* x}\right)\left(I-\Pi_{1, N-1}^{y}\right) u_{1}\right\|_{1}^{2} \\
\leq C \int_{-1}^{1}\left\|\frac{\partial}{\partial y}\left(I-\Pi_{1, N-1}^{y}\right)\left(I-\Pi_{N}^{* x}\right) u_{1}\right\|_{L^{2}(\Lambda)}^{2} d x \\
\quad+C \int_{-1}^{1}\left\|\frac{\partial}{\partial x}\left(I-\Pi_{N}^{* x}\right)\left(I-\Pi_{1, N-1}^{y}\right) u_{1}\right\|_{L^{2}(\Lambda)}^{2} d y .
\end{aligned}
$$

Since $\Pi_{1, N-1}^{y}$ is continuous for the $\mathbf{H}_{0}^{1}(\Lambda)$-norm, and $\Pi_{N}^{* x}$ commutes with differentiation with respect to $y$, we obtain

$$
\int_{-1}^{1}\left\|\frac{\partial}{\partial y}\left(I-\Pi_{1, N-1}^{y}\right)\left(I-\Pi_{N}^{* x}\right) u_{1}\right\|_{L^{2}(\Lambda)}^{2} d x \leq C \int_{-1}^{1}\left\|\left(I-\Pi_{N}^{* x}\right) \frac{\partial u_{1}}{\partial y}\right\|_{L^{2}(\Lambda)}^{2} d x
$$

hence

$$
\begin{gathered}
\int_{-1}^{1}\left\|\frac{\partial}{\partial y}\left(I-\Pi_{1, N-1}^{y}\right)\left(I-\Pi_{N}^{* x}\right) u_{1}\right\|_{L^{2}(\Lambda)}^{2} d x \\
\leq C \int_{-1}^{1}\left\|\left(I-\Pi_{N}^{* x}\right) \frac{\partial u_{1}}{\partial y}\right\|_{L^{2}(\Lambda)}^{2} d y .
\end{gathered}
$$

From (2.26) we deduce that

$$
\int_{-1}^{1}\left\|\frac{\partial}{\partial y}\left(I-\Pi_{1, N-1}^{y}\right)\left(I-\Pi_{N}^{* x}\right) u_{1}\right\|_{L^{2}(\Lambda)}^{2} d x \leq C N^{2-2 \sigma}\|u\|_{\sigma}^{2} \quad \text { for } \sigma \geq 3
$$

The operator $\Pi_{N}^{*}$ is not continuous for the $\mathbf{H}_{0}^{1}(\Lambda)$-norm; by $(2.26)$ we obtain

$$
\begin{gathered}
\int_{-1}^{1}\left\|\frac{\partial}{\partial x}\left(I-\Pi_{N}^{* x}\right)\left(I-\Pi_{1, N-1}^{y}\right) u_{1}\right\|_{L^{2}(\Lambda)}^{2} d y \\
\leq C N^{-2} \int_{-1}^{1}\left\{\left\|\left(I-\Pi_{1, N-1}^{y}\right) \frac{\partial u_{1}}{\partial x}\right\|_{L^{2}(\Lambda)}^{2}\right. \\
\left.+\left\|\left(I-\Pi_{1, N-1}^{y}\right) \frac{\partial^{2} u_{1}}{\partial x^{2}}\right\|_{L^{2}(\Lambda)}^{2}\right\} d y
\end{gathered}
$$

that is,

$$
\begin{gathered}
\int_{-1}^{1}\left\|\frac{\partial}{\partial x}\left(I-\Pi_{N}^{* x}\right)\left(I-\Pi_{1, N-1}^{y}\right) u_{1}\right\|_{L^{2}(\Lambda)}^{2} d y \\
\leq C N^{-2} \int_{-1}^{1}\left\{\left\|\left(I-\Pi_{1, N-1}^{y}\right) \frac{\partial u_{1}}{\partial x}\right\|_{L^{2}(\Lambda)}^{2}\right. \\
\left.+\left\|\left(I-\Pi_{1, N-1}^{y}\right) \frac{\partial^{2} u_{1}}{\partial x^{2}}\right\|_{L^{2}(\Lambda)}^{2}\right\} d x
\end{gathered}
$$


Using (2.4), we deduce

$$
\int_{-1}^{1}\left\|\frac{\partial}{\partial x}\left(I-\Pi_{N}^{* x}\right)\left(I-\Pi_{1, N-1}^{y}\right) u_{1}\right\|_{L^{2}(\Lambda)}^{2} d y \leq C N^{2-2 \sigma}\|u\|_{\sigma}^{2} \quad \text { for } \sigma \geq 3
$$

The estimates (2.36), (2.37) and (2.38) yield

$$
\left\|\left(I-\Pi_{N}^{* x}\right)\left(I-\Pi_{1, N-1}^{y}\right) u_{1}\right\|_{1} \leq C N^{1-\sigma}\|u\|_{\sigma} \quad \text { for } \sigma \geq 3 .
$$

(1b) We now consider the second term in the right-hand side of (2.35). We observe that

$$
\begin{aligned}
\left\|\left(I-\Pi_{N}^{* x}\right) u_{1}\right\|_{1}^{2} \leq & C \int_{-1}^{1}\left\|\frac{\partial}{\partial x}\left(I-\Pi_{N}^{* x}\right) u_{1}\right\|_{L^{2}(\Lambda)}^{2} d y \\
& +C \int_{-1}^{1}\left\|\left(I-\Pi_{N}^{* x}\right) \frac{\partial u_{1}}{\partial y}\right\|_{L^{2}(\Lambda)}^{2} d x
\end{aligned}
$$

and by (2.26) we obtain

$$
\left\|\left(I-\Pi_{N}^{* x}\right) u_{1}\right\|_{1} \leq C N^{1-\sigma}\|u\|_{\sigma} \text { for } \sigma \geq 3 .
$$

(1c) The third term in the right-hand side of (2.35) can be estimated in the same way. Thus, (2.35), (2.39) and (2.40) yield (2.34) for $\nu=1$.

(2) We now prove (2.34) for $\nu=0$. We observe that

$$
\begin{aligned}
\left\|u_{1}-\left(\Pi_{N} \mathbf{u}\right)_{1}\right\|_{0} \leq & \left\|\left(I-\Pi_{N}^{* x}\right)\left(I-\Pi_{1, N-1}^{y}\right) u_{1}\right\|_{0}+\left\|\left(I-\Pi_{N}^{* x}\right) u_{1}\right\|_{0} \\
& +\left\|\left(I-\Pi_{1, N-1}^{y}\right) u_{1}\right\|_{0} .
\end{aligned}
$$

To estimate the first term in the right-hand side of $(2.41)$, we note that

$$
\begin{aligned}
\left\|\left(I-\Pi_{N}^{* x}\right)\left(I-\Pi_{1, N-1}^{y}\right) u_{1}\right\|_{0}^{2} & =\int_{-1}^{1}\left\|\left(I-\Pi_{1, N-1}^{y}\right)\left(I-\Pi_{N}^{* x}\right) u_{1}\right\|_{L^{2}(\Lambda)}^{2} d x \\
& \leq C N^{-2} \int_{-1}^{1}\left\|\left(I-\Pi_{N}^{* x}\right) \frac{\partial u_{1}}{\partial y}\right\|_{L^{2}(\Lambda)}^{2} d x
\end{aligned}
$$

that is,

$$
\begin{aligned}
\left\|\left(I-\Pi_{N}^{* x}\right)\left(I-\Pi_{1, N-1}^{y}\right) u_{1}\right\|_{0}^{2} & \leq C N^{-2} \int_{-1}^{1}\left\|\left(I-\Pi_{N}^{* x}\right) \frac{\partial u_{1}}{\partial y}\right\|_{L^{2}(\Lambda)}^{2} d y \\
& \leq C N^{-2 \sigma}\left\|u_{1}\right\|_{\sigma}^{2} \quad \text { for } \sigma \geq 3 .
\end{aligned}
$$

Along the same lines, we estimate the last two terms in (2.41). Thus the proof of (2.34) for $\nu=0$ is complete.

Finally, using an interpolation argument, we obtain (2.34) for $0 \leq \nu \leq 1$.

Noting now that

$$
\operatorname{div} \Pi_{N} \mathbf{u}=\Pi_{1, N-1}^{x} \Pi_{1, N-1}^{y} \operatorname{div} \mathbf{u},
$$

we obtain (2.33) by the same techniques as used in the proof of (2.32).

3. Projection Operators for Periodic/Nonperiodic Boundary Conditions. In this section we study some approximation properties for a function of three variables which is periodic in two variables. The analysis of projection operators for this type of boundary conditions is useful in studying the spectral approximation of a flow between two parallel planes or between two cylinders. We exhibit the operators we choose, and we only sketch the proofs of the estimates. 
Throughout the section, $\Omega$ is the open set $\Theta^{2} \times \Lambda$ in $\mathbf{R}^{3}$ with $\Theta=(0,2 \pi)$; the generic point in $\Omega$ will be denoted by $\mathbf{x}=(x, y, z)$.

3.1. Cartesian Coordinates. We denote by $\mathbf{C}_{\#}^{\infty}(\overline{\boldsymbol{\theta}})$ the space of infinitely differentiable functions from $\overline{\boldsymbol{\theta}}$ into $\boldsymbol{R}$ which are periodic with period $2 \pi$. For any real number $s$ we introduce the closure $\mathbf{H}_{\#}^{s}(\Theta)$ of $\mathbf{C}_{\#}^{\infty}(\overline{\boldsymbol{\theta}})$ in the space $\mathbf{H}^{\boldsymbol{s}}(\Theta)$; we denote its norm by $\|\cdot\|_{s, \#}$.

Let $\mathbf{C}_{\#}^{\infty}(\bar{\Omega})$ be the space of infinitely differentiable functions from $\bar{\Omega}$ into $\mathbf{R}$ which are periodic with period $2 \pi$ in both directions $x$ and $y$. For any nonnegative integer $m$, we introduce the closure $\mathbf{H}_{\#, \rho}^{m}(\Omega)$ of $\mathbf{C}_{\#}^{\infty}(\bar{\Omega})$ under the norm

$$
\|u\|_{m, \#, \rho}=\left(\sum_{\substack{\mathbf{k} \in \mathbf{N}^{3} \\|\mathbf{k}|_{1} \leq m}} \int_{\Omega}\left|\frac{\partial^{k_{1}+k_{2}+k_{3}}}{\partial x^{k_{1}} \partial y^{k_{2}} \partial z^{k_{3}}} u(\mathbf{x})\right|^{2} \rho(z) d \mathbf{x}\right)^{1 / 2}
$$

where $\rho$ denotes either the Legendre or the Chebyshev weight function.

For any real number $s=m+\alpha, 0<\alpha<1$, we define $\mathbf{H}_{\#, \rho}^{s}(\Omega)$ to be the interpolation space between $\mathbf{H}_{\#, \rho}^{m+1}(\Omega)$ and $\mathbf{H}_{\#, \rho}^{m}(\Omega)$ of index $1-\alpha$; we denote its norm by $\|\cdot\|_{s, \#, \rho}$.

In addition, for any real number $s$ we define $\mathbf{H}_{\#, \rho, 0}^{s}(\Omega)$ to be the closure in $\mathbf{H}_{\#, \rho}^{s}(\Omega)$ of the space of functions of $\mathbf{C}_{\#}^{\infty}(\bar{\Omega})$ which have compact support with respect to the $z$ variable.

For any integers $K$ and $N$ with $K \geq 0$ and $N \geq 2$ we set

$$
\mathbf{V}_{K, N}=\left\{\mathbf{S}_{K}\left(\Theta^{2}\right) \otimes \mathbf{P}_{N}(\Lambda)\right\} \cap \mathbf{H}_{\#, \rho, 0}^{1}(\Omega)
$$

that is,

$$
\mathbf{V}_{K, N}=\left\{u ; \forall \mathbf{x} \in \Omega, u(\mathbf{x})=\left(1-z^{2}\right) \sum_{\substack{\mathbf{k} \in N^{2} \\|\mathbf{k}|_{1} \leq K}} e^{i\left(k_{1} x+k_{2} y\right)} u_{k}(z), u_{k} \in \mathbf{P}_{N-2}(\Lambda)\right\}
$$

Let $Q_{K}: L^{2}(\Theta) \rightarrow \mathbf{S}_{K}(\Theta)$ be the $\mathbf{L}^{2}(\theta)$-orthogonal projection operator over $\mathbf{S}_{K}(\Theta)$. It is well known that the operator $Q_{K}$ commutes with differentiation, i.e., it satisfies

$$
\forall \varphi \in \mathbf{H}_{\#}^{1}(\Theta), \quad Q_{K}\left(\varphi^{\prime}\right)=\left(Q_{K} \varphi\right)^{\prime} .
$$

We recall the following approximation results (see Pasciak [22]):

THEOREM 3.1. For any real numbers $\nu$ and $\sigma$ with $0 \leq \nu \leq \sigma$, there exists a positive constant $C$ independent of $K$ such that

$$
\forall \varphi \in \mathbf{H}_{\#}^{\sigma}(\Theta), \quad\left\|\varphi-Q_{K} \varphi\right\|_{\nu} \leq C K^{\nu-\sigma}\|\varphi\|_{\sigma} .
$$

For any function $u$ in $\mathrm{L}^{2}(\Omega)$, we define

$$
\left(\begin{array}{ll}
\left(Q_{K}^{x} u\right)(\mathbf{x})=\left(Q_{K} u(\cdot, y, z)\right)(x) & \text { a.e. }(y, z) \text { in } \Theta \times \Lambda \\
\left(Q_{K}^{y} u\right)(\mathbf{x})=\left(Q_{K} u(x, \cdot, z)\right)(y) & \text { a.e. }(x, z) \text { in } \Theta \times \Lambda .
\end{array}\right.
$$


From Theorem 3.1 we easily deduce the following corollary:

COROLLARY 3.1. For any real numbers $\nu$ and $\sigma$ with $0 \leq \nu \leq \sigma$, there exists a positive constant $C$ independent of $K$ such that

$$
\forall u \in \mathbf{H}_{\#, \rho}^{\sigma}(\Omega), \quad\left\|u-Q_{K}^{x} u\right\|_{\nu, \#, \rho}+\left\|u-Q_{K}^{y} u\right\|_{\nu, \#, \rho} \leq C K^{\nu-\sigma}\|u\|_{\sigma, \#, \rho} .
$$

Let $r$ be a real number with $r \geq 1$. For any divergence-free function $\mathbf{u}$ of the space $\left(\mathbf{H}_{\#, \rho}^{r}(\Omega)\right)^{3} \cap\left(\mathbf{H}_{\#, \rho, 0}^{1}(\Omega)\right)^{3}$, let $\wp_{r, K, N}^{\text {div }} \mathbf{u}$ be the orthogonal projection of $\mathbf{u}$, for the $\left(\mathbf{H}_{\#, \rho}^{r}(\Omega)\right)^{3}$-inner product, onto the subspace of divergence-free functions of $\left(\mathbf{V}_{K, N}\right)^{3}$.

We can now state the following approximation result:

THEOREM 3.2. Let $r$ be a real number with $r \geq 1$ and $r \notin \mathbb{N}+\sigma_{0}$. For any real number $\sigma$ such that $\sigma \geq r$, there exists a positive constant $C$ independent of both $K$ and $N$ such that

$$
\begin{aligned}
& \forall \mathbf{u} \in\left(\mathbf{H}_{\#, \rho}^{\sigma}(\Omega)\right)^{3} \cap\left(\mathbf{H}_{\#, \rho, 0}^{1}(\Omega)\right)^{3} ; \operatorname{div} \mathbf{u}=0, \\
& \quad\left\|\mathbf{u}-\wp_{r, K, N}^{\operatorname{div}} \mathbf{u}\right\|_{r, \#, \rho} \leq C\left(K^{r-\sigma}+N^{r-\sigma}\right)\|\mathbf{u}\|_{\sigma, \#, \rho} .
\end{aligned}
$$

Proof. Let $\mathbf{u}$ be a divergence-free function in $\left(\mathbf{H}_{\#, \rho}^{\sigma}(\Omega)\right)^{3} \cap\left(\mathbf{H}_{\#, \rho, 0}^{1}(\Omega)\right)^{3}$, and assume first that the real number $\sigma$ is such that $\sigma \geq r+[r]+3$. Consider the function

$$
\mathbf{U}=\mathbf{Q}_{K}^{x} \mathbf{Q}_{K}^{y} \wp_{r+2, N}^{z} \mathbf{u}
$$

where $\wp_{r, N}^{z}$ is the operator introduced in (2.11). Since $\mathbf{Q}_{K}^{x}$ commutes with any derivative, we note, by using the definition of $\wp_{r, N}^{z}$, that $\mathrm{U}$ is a divergence-free element of $\left(\mathbf{V}_{K, N}\right)^{3}$. The inequality (2.18) (where $\wp_{r, N}^{x, \text { div }}$ is replaced by $\mathbf{Q}_{K}^{x}$ and $\wp_{r, N}^{y, \operatorname{div}}$ by $\left.\mathrm{Q}_{K}^{y}\right)$ yields

$$
\|\mathbf{u}-\mathbf{U}\|_{r, \#, \rho} \leq 3\left\|\mathbf{u}-\wp_{r+2, N}^{z} \mathbf{u}\right\|_{r, \#, \rho}+2\left\|\mathbf{u}-\mathbf{Q}_{K}^{y} \mathbf{u}\right\|_{r, \#, \rho}+\left\|\mathbf{u}-\mathbf{Q}_{K}^{x} \mathbf{u}\right\|_{r, \#, \rho} ;
$$

hence, from the definition of $\wp_{r, K, N}^{\mathrm{div}}$, and using Theorem 2.2 and Corollary 3.1, we get

$$
\left\|\mathbf{u}-\wp_{r, K, N}^{\operatorname{div}} \mathbf{u}\right\|_{r, \#, \rho} \leq\|\mathbf{u}-\mathbf{U}\|_{r, \#, \rho} \leq C\left(K^{r-\sigma}+N^{r-\sigma}\right)\|\mathbf{u}\|_{r, \#, \rho} .
$$

In other words, the operator $\mathbf{I}-\wp_{r, K, N}^{\mathrm{div}}$ is continuous from the subspace of divergence-free functions of $\left(\mathbf{H}_{\#, \rho}^{\sigma}(\Omega)\right)^{3} \cap\left(\mathbf{H}_{\#, \rho, 0}^{1}(\Omega)\right)^{3}$ (with $\sigma \geq r+[r]+3$ ) into $\left(\mathbf{H}_{\#, \rho}^{r}(\Omega)\right)^{3}$ with a norm $\leq C\left(K^{r-\sigma}+N^{r-\sigma}\right)$. On the other hand, it is clear that $\mathbf{I}-\wp_{r, K, N}^{\mathrm{div}}$ is continuous from the subspace of divergence-free functions of $\left(\mathbf{H}_{\#, \rho}^{r}(\Omega)\right)^{3} \cap\left(\mathbf{H}_{\#, \rho, 0}^{1}(\Omega)\right)^{3}$ into $\left(\mathbf{H}_{\#, \rho}^{r}(\Omega)\right)^{3}$ with a norm equal to 1 . Thus, Theorem A.1 gives the estimate (3.3) for any real number $\sigma \geq r$, by using an interpolation argument.

As in Section 2, we give a different approach for the case of the Legendre weight. To state the analog of Theorem 2.5, define an operator $\Pi_{K, N}$ as follows:

$$
\forall \mathbf{u} \in\left(\mathbf{H}_{\#}^{2}(\Omega)\right)^{3},\left(\begin{array}{l}
\left(\boldsymbol{\Pi}_{K, N} \mathbf{u}\right)_{1}=Q_{K}^{x} Q_{K}^{y} \Pi_{1, N-1}^{z} u_{1} \\
\left(\boldsymbol{\Pi}_{K, N} \mathbf{u}\right)_{2}=Q_{K}^{x} Q_{K}^{y} \Pi_{1, N-1}^{2} u_{2} \\
\left(\boldsymbol{\Pi}_{K, N} \mathbf{u}\right)_{3}=Q_{K}^{x} Q_{K}^{y} \Pi_{N}^{* z} u_{3}
\end{array}\right.
$$

For any function $\mathbf{u}$ in $\left(\mathbf{H}_{\#}^{2}(\Omega)\right)^{3} \cap\left(\mathbf{H}_{\#, 0}^{1}(\Omega)\right)^{3}$, the function $\boldsymbol{\Pi}_{K, N} \mathbf{u}$ belongs to $\left(\mathbf{V}_{K, N}\right)^{3}$. Moreover, the divergence of $\boldsymbol{\Pi}_{K, N} \mathbf{u}$ is given by

$$
\operatorname{div} \Pi_{K, N} \mathbf{u}=Q_{K}^{x} Q_{K}^{y} \Pi_{1, N-1}^{z} \operatorname{div} \mathbf{u} ;
$$

hence, if $\mathbf{u}$ is divergence-free, so is $\Pi_{K, N} \mathbf{u}$. 
The proof of the following estimate is analogous to the proof of Theorem 2.5.

THEOREM 3.3. For any real numbers $\nu$ and $\sigma$ with $0 \leq \nu \leq 1$ and $\sigma \geq 3$, there exists a positive constant $C$ independent of both $K$ and $N$ such that

$$
\begin{aligned}
& \forall \mathbf{u} \in\left(\mathbf{H}_{\#}^{\sigma}(\Omega)\right)^{3} \cap\left(\mathbf{H}_{\#, 0}^{1}(\Omega)\right)^{3}, \\
& \qquad \mathbf{u}-\mathbf{\Pi}_{K, N} \mathbf{u}\left\|_{\nu, \#} \leq C\left(K^{\nu-\sigma}+N^{\nu-\sigma}\right)\right\| \mathbf{u} \|_{\sigma, \#}
\end{aligned}
$$

and

(3.5) $\forall \mathbf{u} \in\left(\mathbf{H}_{\#}^{\sigma}(\Omega)\right)^{3} \cap\left(\mathbf{H}_{\#, 0}^{1}(\Omega)\right)^{3}$,

$$
\left\|\operatorname{div} \mathbf{u}-\operatorname{div} \Pi_{K, N} \mathbf{u}\right\|_{\nu, \#} \leq C\left(K^{\nu+1-\sigma}+N^{\nu+1-\sigma}\right)\|\operatorname{div} \mathbf{u}\|_{\sigma-1, \#} .
$$

3.2. Cylindrical Coordinates. Let $R_{0}, R_{1}$ and $L$ be three real numbers such that $0<R_{0}<R_{1}$ and $L>0$. In this section we set $\tilde{\Omega}=\left(R_{0}, R_{1}\right) \times(0,2) \times(-L, L)$, and the generic point in $\tilde{\Omega}$ will be denoted by $\tilde{\mathbf{x}}=(R, \theta, Z)$. We consider functions which are periodic in both directions $\theta$ and $Z$. We let $\tilde{\rho}:\left(R_{0}, R_{1}\right) \rightarrow \mathbb{P}$ be either the Legendre weight function defined by $\tilde{\rho}(R)=1$ or the Chebyshev weight function defined by $\tilde{\rho}(R)=\left(1-\left[\left(2 R-R_{0}-R_{1}\right) /\left(R_{1}-R_{0}\right)\right]^{2}\right)^{-1 / 2}$.

The definitions of the spaces $\mathbf{H}_{\#, \tilde{\rho}}^{s}(\tilde{\Omega})$ and $\mathbf{H}_{\#, \tilde{\rho}, 0}^{s}(\tilde{\Omega})$ are analogous to those of the spaces $\mathbf{H}_{\#, \rho}^{s}(\Omega)$ and $\mathbf{H}_{\#, \rho, 0}^{s}(\Omega)$.

For any function $\tilde{\mathbf{u}}=\left(\tilde{u}_{R}, \tilde{u}_{\theta}, \tilde{u}_{Z}\right)$ from $\tilde{\Omega}$ into $\mathbb{R}^{3}$, the divergence of $\tilde{\mathbf{u}}$ is defined by

$$
\operatorname{div} \tilde{\mathbf{u}}=\frac{1}{R} \frac{\partial\left(R \tilde{u}_{R}\right)}{\partial R}+\frac{1}{R} \frac{\partial \tilde{u}_{\theta}}{\partial \theta}+\frac{\partial \tilde{u}_{Z}}{\partial Z} .
$$

For any integers $K$ and $N$ with $K \geq 0$ and $N \geq 2$, we define the space $\tilde{\mathbf{V}}_{K, N}$ as follows:

$$
\begin{aligned}
& \tilde{\mathbf{V}}_{K, N}=\left\{\tilde{u} ; \forall \tilde{\mathbf{x}} \in \tilde{\Omega}, \tilde{u}(\tilde{\mathbf{x}})=\sum_{\substack{\mathbf{k} \in N^{2} \\
|\mathbf{k}|_{1} \leq K}} \tilde{u}_{k}(R) e^{i\left(k_{1} \theta+k_{2} Z \pi / L\right)},\right. \\
& \left.\tilde{u}_{k} \in \mathbf{P}_{N}\left(\left[R_{0}, R_{1}\right]\right), \tilde{u}_{k}\left(R_{0}\right)=\tilde{u}_{k}\left(R_{1}\right)=0\right\} .
\end{aligned}
$$

Consider the affine change of variables

$$
x=\frac{\pi}{L} Z, \quad y=\theta, \quad z=\frac{2 R-R_{0}-R_{1}}{R_{1}-R_{0}}
$$

which maps $\tilde{\Omega}$ onto $\Omega$.

For any function $\tilde{\mathbf{u}}: \tilde{\Omega} \rightarrow \mathbb{R}^{3}$ we define $\mathbf{u}: \Omega \rightarrow \mathbb{R}^{3}$ as follows:

$$
u_{1}(\mathbf{x})=\frac{\pi}{L} \tilde{u}_{Z}(\tilde{\mathbf{x}}), \quad u_{2}(\mathbf{x})=\frac{2}{R_{1}-R_{0}} \tilde{u}_{\theta}(\tilde{\mathbf{x}}), \quad u_{3}(\mathbf{x})=\frac{2}{R_{1}-R_{0}} \tilde{u}_{R}(\tilde{\mathbf{x}}) .
$$

Letting

$$
\left(\begin{array}{l}
\alpha=-\frac{R_{0}+R_{1}}{R_{1}-R_{0}} \\
\forall \mathbf{x} \in \Omega, \quad a(\mathbf{x})=z-\alpha \\
D \mathbf{u}=\frac{\partial u_{1}}{\partial x}+a^{-1} \frac{\partial u_{2}}{\partial y}+a^{-1} \frac{\partial\left\{a u_{3}\right\}}{\partial z}
\end{array}\right.
$$


we have

$$
\operatorname{div} \tilde{\mathbf{u}}(\tilde{\mathbf{x}})=D \mathbf{u}(\mathbf{x})
$$

The following lemma will be useful in the sequel.

LEMMA 3.1. Let $b$ be a strictly positive function in $\mathbf{C}^{\infty}(\bar{\Lambda})$. Let $\Pi_{N}$ be an operator such that for any real numbers $\nu$ and $\sigma$ with $0 \leq \nu \leq \sigma$ there exists a positive constant $C$ independent of $N$ such that

$$
\forall u \in \mathbf{H}_{\rho}^{\sigma}(\Lambda), \quad\left\|u-\Pi_{N} u\right\|_{\nu, \rho} \leq C N^{\nu-\sigma}\|u\|_{\sigma, \rho} .
$$

Setting

$$
\forall u \in \mathbf{H}_{\rho}^{\sigma}(\Lambda), \quad\left(\Pi_{N}\right)_{b} u=b^{-1} \Pi_{N}(b u),
$$

for any real numbers $\nu$ and $\sigma$ with $0 \leq \nu \leq \sigma$ there exists a positive constant $C^{\prime}$ independent of $N$ such that

$$
\forall u \in \mathbf{H}_{\rho}^{\sigma}(\Lambda), \quad\left\|u-\left(\Pi_{N}\right)_{b} u\right\|_{\nu, \rho} \leq C^{\prime} N^{\nu-\sigma}\|u\|_{\sigma, \rho} .
$$

Proof. If $\nu$ is an integer, it is clear that the mapping $u \rightarrow\|b u\|_{\nu, \rho}$ is a norm on $\mathbf{H}_{\rho}^{\nu}(\Lambda)$ which is equivalent to the norm $\|\cdot\|_{\nu, \rho}$. If $\nu$ is a real number, we know that there exist four positive constants $C_{1}, C_{2}, C_{3}$ and $C_{4}$ such that

$$
\left(\begin{array}{l}
\forall u \in \mathbf{H}_{\rho}^{[\nu]}(\Lambda), \quad C_{1}\|b u\|_{[\nu], \rho} \leq\|b u\|_{[\nu], \rho} \leq C_{2}\|b u\|_{[\nu], \rho}, \\
\forall u \in \mathbf{H}_{\rho}^{[\nu+1]}(\Lambda), \quad C_{3}\|b u\|_{[\nu+1], \rho} \leq\|b u\|_{[\nu+1], \rho} \leq C_{4}\|b u\|_{[\nu+1], \rho} .
\end{array}\right.
$$

Hence, by using an interpolation argument, we deduce that the mapping $u \rightarrow$ $\|b u\|_{\nu, \rho}$ is a norm on $\mathbf{H}_{\rho}^{\nu}(\Lambda)$ which is equivalent to the norm $\|\cdot\|_{\nu, \rho}$. In the same way we prove that for any real number $\nu$ the mapping $u \rightarrow\left\|b^{-1} u\right\|_{\nu, \rho}$ is a norm on $\mathbf{H}_{\rho}^{\nu}(\Lambda)$ which is equivalent to the norm $\|\cdot\|_{\nu, \rho}$. Thus $(3.13)$ is a direct consequence of (3.12).

Let $r$ be a real number with $r \geq 1$. For any divergence-free function $\tilde{\mathbf{u}}$ of the space $\left(\mathbf{H}_{\#, \tilde{\rho}}^{r}(\tilde{\Omega})\right)^{3} \cap\left(\mathbf{H}_{\#, \tilde{\rho}, 0}^{1}(\tilde{\Omega})\right)^{3}$, let $\tilde{\wp}_{r, K, N}^{\text {div }} \tilde{\mathbf{u}}$ be the orthogonal projection of $\tilde{\mathbf{u}}$ for the $\left(\mathbf{H}_{\#, \tilde{\rho}}^{r}(\tilde{\Omega})\right)^{3}$-inner product on the subspace of divergence-free functions of $\left(\tilde{\mathbf{V}}_{K, N}\right)^{3}$.

We have the following approximation result:

THEOREM 3.4. Let $r$ be a real number with $r \geq 1$ and $r \notin \mathbf{N}+\sigma_{0}$. For any real number $\sigma$ such that $\sigma \geq r$, there exists a positive constant $C$ independent of both $K$ and $N$ such that

$$
\begin{aligned}
\forall \tilde{\mathbf{u}} \in\left(\mathbf{H}_{\#, \tilde{\rho}}^{\sigma}(\tilde{\Omega})\right)^{3} \cap\left(\mathbf{H}_{\#, \tilde{\rho}, 0}^{1}(\tilde{\Omega})\right)^{3}, \operatorname{div} \tilde{\mathbf{u}}=0, \\
\\
\quad\left\|\tilde{\mathbf{u}}-\tilde{\varphi}_{r, K, N}^{\operatorname{div}} \tilde{\mathbf{u}}\right\|_{r, \#, \tilde{\rho}} \leq C\left(K^{r-\sigma}+N^{r-\sigma}\right)\|\tilde{\mathbf{u}}\|_{\sigma, \#, \tilde{\rho}}
\end{aligned}
$$

Proof. Let $\tilde{\mathbf{u}}$ be a divergence-free function which belongs to $\left(\mathbf{H}_{\#, \tilde{\rho}}^{\sigma}(\tilde{\Omega})\right)^{3} \cap$ $\left(\mathbf{H}_{\#, \tilde{\rho}, 0}^{1}(\tilde{\Omega})\right)^{3}$. We use the change of variables (3.7) and the function $\mathbf{u}$ defined by (3.8). Since the change of variables is a $C^{\infty}$-diffeomorphism $\tilde{\Omega} \rightarrow \Omega$, the function $\mathbf{u}$ belongs to the space $\left(\mathbf{H}_{\#, \rho}^{\sigma}(\Omega)\right)^{3} \cap\left(\mathbf{H}_{\#, \rho, 0}^{1}(\Omega)\right)^{3}$. Moreover, by $(3.10), \mathbf{u}$ is such that $D \mathbf{u}=0$. 
Denote by $\wp_{r, K, N}^{D} \mathbf{u}$ the orthogonal projection of $\mathbf{u}$, for the $\left(\mathbf{H}_{\#, \rho}^{r}(\Omega)\right)^{3}$-inner product, on the subspace of the functions $\mathbf{v}$ of $\left(\mathbf{V}_{K, N}\right)^{3}$ such that $D \mathbf{v}=0$. Hence, in order to prove (3.14), we are led to derive

$$
\left\|\mathbf{u}-\wp_{r, K, N}^{D} \mathbf{u}\right\|_{r, \#, \rho} \leq C\left(K^{r-\sigma}+N^{r-\sigma}\right)\|\mathbf{u}\|_{\sigma, \#, \rho}
$$

for a positive constant $C$ independent of $K, N$ and $\mathbf{u}$.

We get the estimate (3.15) following the technique of the proof of Theorem 3.2: we begin by proving it for any real number $\sigma$ such that $\sigma \geq r+[r]+3$; here we replace the operator $\wp_{r+2, N}^{z}$ by an operator $\mathfrak{R}_{r+2, N}^{z}$ which takes into account the new structure of the divergence operator.

(1) For any real number $q$ such that $q \geq 1$ and $q \notin \mathbb{N}+\sigma_{0}$, we now define the operator $\mathfrak{R}_{q, N}^{z}$.

For any function $\mathbf{v}$ in $\left(\mathbf{H}_{\#, \rho}^{q}(\Omega)\right)^{3}$, first introduce the functions:

$$
\psi_{1}(\mathbf{x})=-\int_{-1}^{z} v_{2}(x, y, t) d t \quad \text { and } \quad \psi_{2}(\mathbf{x})=\int_{-1}^{z}(t-\alpha) v_{1}(x, y, t) d t
$$

Recalling (3.9) and (3.12), we then set

$$
\left(\begin{array}{l}
\left(\mathfrak{R}_{q, N}^{z} \mathbf{v}\right)_{1}=a^{-1} \frac{\partial}{\partial z}\left[\left(\Pi_{q, N-1}^{z}\right)_{a^{-2}}\left(\psi_{2}\right)\right] \\
\left(\Re_{q, N}^{z} \mathbf{v}\right)_{2}=-\frac{\partial}{\partial z}\left[\left(\Pi_{q, N-1}^{z}\right)_{a^{-2}}\left(\psi_{1}\right)\right] \\
\left(\mathfrak{R}_{q, N}^{z} \mathbf{v}\right)_{3}=\left(\Pi_{q, N-1}^{z}\right)_{a^{-1}}\left(v_{3}\right) .
\end{array}\right.
$$

(1a) It is easy to see that the function $\mathfrak{R}_{q, N}^{z} \mathbf{v}$ is polynomial in $z$ of degree $\leq N$.

(1b) Using Lemma 3.1 and the technique of the proof of Theorem 2.2, we prove the following result: if $q$ is a real number such that $q \geq 1$ and $q \notin \mathbf{N}+\sigma_{0}$, then for any integer $\nu$ such that $0 \leq \nu \leq q-1$ and for any real number $s$ such that $s \geq q+\nu$, there exists a positive constant $C$ independent of $N$ such that

$$
\forall \mathbf{v} \in\left(\mathbf{H}_{\#, \rho}^{s}(\Omega)\right)^{3}, \quad\left\|\mathbf{v}-\mathfrak{R}_{q, N}^{z} \mathbf{v}\right\|_{\nu, \#, \rho} \leq C N^{\nu-s}\|\mathbf{v}\|_{s, \#, \rho}
$$

Moreover, the preceding estimate is still valid if $q, \nu$ and $s$ are real numbers such that $q \geq 2, q \notin \mathbf{N}+\sigma_{0}, 0 \leq \nu,[\nu] \leq q-2$ and $s \geq q+1+[\nu]$.

(1c) Let now $q$ be a real number such that $q \geq 2$, and $\mathbf{v}$ be an element of the space $\left(\mathbf{H}_{\#, \rho}^{q+1}(\Omega)\right)^{3} \cap\left(\mathbf{H}_{\#, \rho, 0}^{1}(\Omega)\right)^{3}$. We prove that $\mathfrak{R}_{q, N}^{z} \mathbf{v}$ belongs to $\left(\mathbf{H}_{\#, \rho, 0}^{1}(\Omega)\right)^{3}$. In view of the preceding estimate, we know that $\mathfrak{R}_{q, N}^{z} \mathbf{v}$ belongs to $\left(\mathbf{H}_{\#, \rho}^{1}(\Omega)\right)^{3}$; hence it is sufficient to prove that $\mathfrak{R}_{q, N}^{z} \mathbf{v}$ is such that

$$
\left(\Re_{q, N}^{z} \mathbf{v}\right)(x, y, \pm 1)=0 \text { a.e. }(x, y) \text { in } \Theta^{2} \text {. }
$$

We first compute $\left(\mathfrak{R}_{q, N}^{z} \mathbf{v}\right)_{2}(\mathbf{x})$ :

$$
\begin{aligned}
\left(\mathfrak{R}_{q, N}^{z} \mathbf{v}\right)_{2}(\mathbf{x})= & 2 a(\mathbf{x})\left(\Pi_{q, N-1}^{z}\right)\left(\frac{\int_{-1}^{z} \mathbf{v}_{2}(x, y, t) d t}{a(\mathbf{x})^{2}}\right) \\
& +a(\mathbf{x})^{2} \frac{\partial}{\partial z}\left\{\left(\Pi_{q, N-1}^{z}\right)\left(\frac{\int_{-1}^{z} \mathbf{v}_{2}(x, y, t) d t}{a(\mathbf{x})^{2}}\right)\right\} \quad \text { a.e. } \mathbf{x} \text { in } \Theta^{2} .
\end{aligned}
$$

Moreover, according to Corollary 2.1, for any function $v$ in $\left(\mathbf{H}_{\#, \rho}^{2}(\Omega)\right)^{3}$ we have

$$
\forall j \in\{0,1\}, \quad \frac{\partial^{j}}{\partial z^{j}}\left(\Pi_{q, N-1}^{z} v\right)(x, y, \pm 1)=\frac{\partial^{j} v}{\partial z^{j}}(x, y, \pm 1) \quad \text { a.e. }(x, y) \text { in } \Theta^{2}
$$


Thus we deduce

$$
\begin{aligned}
& \forall z= \pm 1, \quad\left(\mathfrak{R}_{q, N}^{z} \mathbf{v}\right)_{2}(x, y, z)= 2 a(\mathbf{x})\left(\frac{\int_{-1}^{z} \mathbf{v}_{2}(x, y, t) d t}{a(\mathbf{x})^{2}}\right) \\
&+a(\mathbf{x})^{2} \frac{\partial}{\partial z}\left(\frac{\int_{-1}^{z} \mathbf{v}_{2}(x, y, t) d t}{a(\mathbf{x})^{2}}\right) \\
& \text { a.e. }(x, y) \text { in } \Theta^{2}
\end{aligned}
$$

that is,

$$
\left(\Re_{q, N}^{z} \mathbf{v}\right)_{2}(x, y, \pm 1)=\mathbf{v}_{2}(x, y, \pm 1)=0 \quad \text { a.e. }(x, y) \text { in } \Theta^{2} .
$$

In the same way we finally prove that $\left(\Re_{q, N}^{z} \mathbf{v}\right)$ belongs to $\left(\mathbf{H}_{\#, \rho, 0}^{1}(\Omega)\right)^{3}$.

(1d) We now prove that for any function $\mathbf{v}$ such that $D \mathbf{v}=0, \Re_{q, N}^{z} \mathbf{v}$ is such that $D \Re_{q, N}^{z} \mathbf{v}=0$.

Since the operators $\left(\Pi_{q, N-2}^{z}\right)_{a^{-1}}$ and $\left(\Pi_{q, N-2}^{z}\right)_{a^{-2}}$ commute with differentiation with respect to $x$ and $y$, we have

$$
\begin{aligned}
D \mathfrak{R}_{q, N}^{z} \mathbf{v} & =\frac{\partial\left(\mathfrak{R}_{q, N}^{z} \mathbf{v}\right)_{1}}{\partial x}+a^{-1} \frac{\partial\left(\mathfrak{R}_{q, N}^{z} \mathbf{v}\right)_{2}}{\partial y}+a^{-1} \frac{\partial\left\{a\left(\mathfrak{R}_{q, N}^{z} \mathbf{v}_{3}\right)\right\}}{\partial z} \\
& =a^{-1} \frac{\partial}{\partial z}\left(\left(\Pi_{q, N-2}^{z}\right)_{a^{-2}}\left(\frac{\partial \psi_{2}}{\partial x}-\frac{\partial \psi_{1}}{\partial y}\right)+a\left(\Pi_{q, N-2}^{z}\right)_{a^{-1}} \mathbf{v}_{3}\right)
\end{aligned}
$$

Since the function $\mathbf{v}$ is such that $D \mathbf{v}=0$, we have

$$
v_{3}=a^{-1}\left(\frac{\partial \varphi_{1}}{\partial y}-\frac{\partial \varphi_{2}}{\partial x}\right) ;
$$

hence, using (3.12), we conclude that $D \Re_{q, N}^{z} \mathbf{v}=0$.

(2) Using the operator $\mathfrak{R}_{r+2, N}^{z}$ just defined, and proceeding as in the proof of Theorem 3.2, we obtain (3.15). This completes the proof of the theorem.

The analog of Theorem 2.5 reads as follows:

THEOREM 3.5. There exists an operator $\tilde{\Pi}_{K, N}:\left(\mathbf{H}_{\#}^{2}(\tilde{\Omega})\right)^{3} \cap\left(H_{\#, 0}^{1}(\tilde{\Omega})\right)^{3} \rightarrow$ $\left(\tilde{\mathbf{V}}_{K, N}\right)^{3}$ such that for any real numbers $\nu$ and $\sigma$ with $0 \leq \nu \leq 1$ and $\sigma \geq 3$ there exists a positive constant $C$ independent of both $K$ and $N$ such that

$$
\begin{aligned}
& \forall \tilde{\mathbf{u}} \in\left(\mathbf{H}_{\#}^{\sigma}(\tilde{\Omega})\right)^{3} \cap\left(\mathbf{H}_{\#, 0}^{1}(\tilde{\Omega})\right)^{3}, \\
& \qquad \tilde{\mathbf{u}}-\tilde{\Pi}_{K, N} \tilde{\mathbf{u}}\left\|_{\nu, \#} \leq C\left(K^{\nu-\sigma}+N^{\nu-\sigma}\right)\right\| \tilde{\mathbf{u}} \|_{\sigma, \#}
\end{aligned}
$$

and

$$
\begin{aligned}
& \forall \tilde{\mathbf{u}} \in\left(\mathbf{H}_{\#}^{\sigma}(\tilde{\Omega})\right)^{3} \cap\left(\mathbf{H}_{\#, 0}^{1}(\tilde{\Omega})\right)^{3}, \\
& \quad\left\|\operatorname{div} \tilde{\mathbf{u}}-\operatorname{div} \tilde{\boldsymbol{\Pi}}_{K, N} \tilde{\mathbf{u}}\right\|_{\nu, \#} \leq C\left(K^{\nu+1-\sigma}+N^{\nu+1-\sigma}\right)\|\operatorname{div} \tilde{\mathbf{u}}\|_{\sigma-1, \#} .
\end{aligned}
$$

Proof. As in the proof of Theorem 3.4, we are led to prove that there exists an operator $\boldsymbol{\Pi}_{K, N}:\left(\mathbf{H}_{\#}^{2}(\Omega)\right)^{3} \cap\left(\mathbf{H}_{\#, 0}^{1}(\Omega)\right)^{3} \rightarrow\left(\mathbf{V}_{K, N}\right)^{3}$ such that for any real numbers $\nu$ and $\sigma$ with $0 \leq \nu \leq 1$ and $\sigma \geq 2$ there exists a positive constant $C$ independent of both $K$ and $N$ such that

$$
\begin{aligned}
\forall \mathbf{u} \in\left(\mathbf{H}_{\#}^{\sigma}(\Omega)\right)^{3} \cap\left(\mathbf{H}_{\#, 0}^{1}(\Omega)\right)^{3} \\
\qquad\left\|\mathbf{u}-\mathbf{\Pi}_{K, N} \mathbf{u}\right\|_{\nu, \#} \leq C\left(K^{\nu-\sigma}+N^{\nu-\sigma}\right)\|\mathbf{u}\|_{\sigma, \#}
\end{aligned}
$$


and

$$
\begin{aligned}
& \forall \mathbf{u} \in\left(\mathbf{H}_{\#}^{\sigma}(\Omega)\right)^{3} \cap\left(\mathbf{H}_{\#, 0}^{1}(\Omega)\right)^{3} \\
& \quad\left\|D \mathbf{u}-D \mathbf{\Pi}_{K, N} \mathbf{u}\right\|_{\nu, \#} \leq C\left(K^{\nu+1-\sigma}+N^{\nu+1-\sigma}\right)\|\operatorname{div} \mathbf{u}\|_{\sigma-1, \#}
\end{aligned}
$$

Recalling the definitions (2.6), (3.1), (3.9) and (3.10), we introduce two operators $T_{N}$ and $\Pi_{K, N}$ as follows:

$$
\begin{gathered}
\forall u \in \mathbf{H}_{\#}^{2}(\Omega), \forall \mathbf{x} \in \Omega, \quad\left(T_{N} u\right)(\mathbf{x})=\frac{\partial}{\partial z}\left[\left(\Pi_{2, N-2}^{z}\right)_{a^{-2}}\left(\int_{-1}^{z} u(x, y, t) d t\right)\right], \\
\forall \mathbf{u} \in\left(\mathbf{H}_{\#}^{2}(\Omega)\right)^{3}, \quad \begin{array}{l}
\left(\Pi_{K, N} \mathbf{u}\right)_{1}=Q_{K}^{x} Q_{K}^{y}\left(T_{N}\right)_{a} u_{1}, \\
\left(\Pi_{K, N} \mathbf{u}\right)_{2}=Q_{K}^{x} Q_{K}^{y} T_{N} u_{2}, \\
\left(\Pi_{K, N} \mathbf{u}\right)_{3}=Q_{K}^{x} Q_{K}^{y}\left(\Pi_{2, N}^{z}\right)_{a^{-1}} u_{3} .
\end{array}
\end{gathered}
$$

(1) Arguing as in the proof of Theorem 3.4, we prove that for any element $\mathbf{u}$ of the space $\left(\mathbf{H}_{\#}^{2}(\Omega)\right)^{3} \cap\left(\mathbf{H}_{\#, 0}^{1}(\Omega)\right)^{3}, \boldsymbol{\Pi}_{K, N} \mathbf{u}$ belongs to $\left(\mathbf{V}_{K, N}\right)^{3}$.

(2) Let now $\nu$ and $\sigma$ be two real numbers such that $0 \leq \nu \leq 1, \sigma \geq 2$, and let $\mathbf{u}$ be an element of $\left(\mathbf{H}_{\#}^{\sigma}(\Omega)\right)^{3} \cap\left(\mathbf{H}_{\#, 0}^{1}(\Omega)\right)^{3}$. In order to prove the estimates (3.18) and (3.19), we follow the proof of Theorem 2.5. Since we have

$$
D \Pi_{K, N} \mathbf{u}=Q_{K}^{x} Q_{K}^{y}\left(T_{N}\right)_{a} D \mathbf{u}
$$

it is sufficient to prove that for any function $u$ in $\mathbf{H}_{\#}^{\sigma}(\Omega)$ we have

$$
\left\|u-T_{N} u\right\|_{\nu, \#} \leq C N^{\nu-\sigma}\|u\|_{\sigma, \#}, \quad\left\|u-\left(T_{N} u\right)_{a}\right\|_{\nu, \#} \leq C N^{\nu-\sigma}\|u\|_{\sigma, \#}
$$

and

$$
\left\|u-\left(\Pi_{2, N}^{z}\right)_{s^{-1}} u\right\|_{\nu, \#} \leq C N^{\nu-\sigma}\|u\|_{\sigma, \#},
$$

where $C$ is a positive constant independent of both $N$ and $u$.

We follow the proof of Theorem 2.2: In view of Corollary 2.1 and Lemma 3.1 it is sufficient to prove that for any real numbers $\lambda$ and $\mu$ with $0 \leq \lambda \leq 1 \leq \mu$ there exists a positive constant $C$ such that

$$
\forall \varphi \in \mathbf{H}_{\mu}(\Lambda), \quad\left\|\varphi-\left[\left(\Pi_{2, N}^{z}\right)_{a^{-2}}\left(\int_{-1}^{z} \varphi(t) d t\right)\right]^{\prime}\right\|_{\lambda} \leq C N^{\lambda-\mu}\|\varphi\|_{\mu} .
$$

This estimate follows from Corollary 2.1 and Lemma 3.1. Thus, (3.18) and (3.19) are proved. This completes the proof of Theorem 3.5.

4. An Application. For some applications of our results we refer the reader to F. Pasquarelli, A. Quarteroni and G. Sacchi Landriani [23]. In this section we consider the Legendre spectral approximation of the Stokes system between two cylinders. This approximation scheme has been introduced and implemented by $\mathrm{N}$. Mac Giolla Mhuiris (see [16] and the references therein for other methods of this type). Using the notations of Section 3, the variational formulation of the Stokes problem between two cylinders is as follows: given a function $\tilde{\mathbf{f}}$ in $\left(\mathbf{L}^{2}(\tilde{\Omega})\right)^{3}$, find $\tilde{\mathbf{u}}$ in the space $\left(\left(\mathbf{H}_{\#}^{2}(\tilde{\Omega})\right)^{3} \cap\left(\mathbf{H}_{\#, 0}^{1}(\tilde{\Omega})\right)^{3}\right.$ such that

$$
\left(\begin{array}{l}
\forall \tilde{\mathbf{v}} \in\left(\mathbf{L}^{2}(\tilde{\Omega})\right)^{3} ; \operatorname{div} \tilde{\mathbf{v}}=0,-\int_{\tilde{\Omega}} \tilde{\mathbf{v}} \Delta \tilde{\mathbf{u}} R d R d \theta d Z=\int_{\tilde{\Omega}} \tilde{\mathbf{f}} \tilde{\mathbf{v}} R d R d \theta d Z \\
\operatorname{div} \tilde{\mathbf{u}}=0 \text { in } \tilde{\Omega} .
\end{array}\right.
$$


Here, the operators div and $\Delta$ are defined by

$$
\forall \tilde{\mathbf{v}}=\left(\tilde{v}_{R}, \tilde{v}_{\theta}, \tilde{v}_{Z}\right), \quad\left(\begin{array}{l}
\operatorname{div} \tilde{\mathbf{v}}=\frac{1}{R} \frac{\partial\left(R \tilde{v}_{R}\right)}{\partial R}+\frac{1}{R} \frac{\partial \tilde{v}_{\theta}}{\partial \theta}+\frac{\partial \tilde{v}_{Z}}{\partial Z} \\
\Delta \tilde{\mathbf{v}}=\frac{1}{R} \frac{\partial}{\partial R}\left(R \frac{\partial \tilde{\mathbf{v}}}{\partial R}\right)+\frac{1}{R^{2}} \frac{\partial^{2} \tilde{\mathbf{v}}}{\partial \theta^{2}}+\frac{\partial^{2} \tilde{\mathbf{v}}}{\partial Z^{2}}
\end{array}\right.
$$

We consider the following spectral approximation of problem (4.1): find $\tilde{\mathbf{u}}_{K, N}$ in $\left(\tilde{\mathbf{V}}_{K, N}\right)^{3}$ such that

$$
\left(\begin{array}{l}
\forall \tilde{\mathbf{v}}_{K, N} \in\left(\tilde{\mathbf{V}}_{K, N}\right)^{3} ; \operatorname{div} \tilde{\mathbf{v}}_{K, N}=0 \\
\quad-\int_{\tilde{\Omega}} \tilde{\mathbf{v}}_{K, N} \Delta \tilde{\mathbf{u}}_{K, N} R d R d \theta d Z=\int_{\tilde{\Omega}} \tilde{\mathbf{f}}^{\mathbf{\mathbf { v }}} \tilde{K}_{K, N} R d R d \theta d Z \\
\operatorname{div} \tilde{\mathbf{u}}_{K, N}=0 \quad \operatorname{in} \tilde{\Omega} .
\end{array}\right.
$$

We have the following convergence result for the solution of problem (4.2).

THEOREM 4.1. Let $\sigma \geq 1$ be a real number. If the solution $\tilde{\mathbf{u}}$ of problem (4.1) belongs to $\left(H_{\#}^{\sigma}(\tilde{\Omega})\right)^{3}$, there exists a positive constant $C$ independent of $\tilde{\mathbf{u}}, K$ and $N$ such that

$$
\left\|\tilde{\mathbf{u}}-\tilde{\mathbf{u}}_{K, N}\right\|_{1, \#} \leq C\left(K^{1-\sigma}+N^{1-\sigma}\right)\|\tilde{\mathbf{u}}\|_{\sigma, \#}
$$

Proof. Let $\tilde{\emptyset}_{1, K, N}^{\text {div }} \tilde{\mathbf{u}}$ be the orthogonal projection of $\tilde{\mathbf{u}}$ for the inner product of $\left(\mathbf{H}_{\#}^{\mathbf{1}}(\tilde{\Omega})\right)^{3}$ on the subspace of divergence-free functions of $\left(\tilde{\mathbf{V}}_{K, N}\right)^{3}$. Using (4.1) and (4.2), we deduce

$$
\begin{gathered}
\forall \tilde{\mathbf{v}}_{K, N} \in\left(\tilde{\mathbf{V}}_{K, N}\right)^{3} ; \operatorname{div} \tilde{\mathbf{v}}_{K, N}=0, \int_{\tilde{\Omega}} \tilde{\mathbf{v}}_{K, N} \Delta\left(\tilde{\mathbf{u}}_{K, N}-\tilde{\wp}_{1, K, N}^{\operatorname{div}} \tilde{\mathbf{u}}\right) R d R d \theta d Z \\
=\int_{\tilde{\Omega}} \tilde{\mathbf{v}}_{K, N} \Delta\left(\tilde{\mathbf{u}}-\tilde{\wp}_{1, K, N}^{\text {div }} \tilde{\mathbf{u}}\right) R d R d \theta d Z
\end{gathered}
$$

Choosing $\tilde{\mathbf{v}}_{K, N}=\tilde{\mathbf{u}}_{K, N}-\tilde{\wp}_{1, K, N}^{\text {div }} \tilde{\mathbf{u}}$ in (4.4) and using the following rule of integration by parts:

$$
-\int_{\tilde{\Omega}} \tilde{\mathbf{v}} \Delta \tilde{\mathbf{w}} R d R d \theta d Z=\int_{\tilde{\Omega}} \operatorname{grad} \tilde{\mathbf{v}} \cdot \operatorname{grad} \tilde{\mathbf{u}} R d R d \theta d Z
$$

we obtain

$$
\begin{aligned}
\int_{\tilde{\Omega}}\left|\operatorname{grad}\left(\tilde{\mathbf{u}}_{K, N}-\tilde{\wp}_{1, K, N}^{\operatorname{div}} \tilde{\mathbf{u}}\right)\right|^{2} R d R d \theta d Z & \\
& =\int_{\tilde{\Omega}} \operatorname{grad}\left(\tilde{\mathbf{u}}_{K, N}-\tilde{\wp}_{1, K, N}^{\text {div }} \tilde{\mathbf{u}}\right) \cdot \operatorname{grad}\left(\tilde{\wp}_{1, K, N}^{\operatorname{div}} \tilde{\mathbf{u}}\right) R d R d \theta d Z .
\end{aligned}
$$

By using the Cauchy-Schwarz inequality, the preceding formula yields

$$
\int_{\tilde{\Omega}}\left|\operatorname{grad}\left(\tilde{\mathbf{u}}_{K, N}-\tilde{\wp}_{1, K, N}^{\mathrm{div}} \tilde{\mathbf{u}}\right)\right|^{2} R d R d \theta d Z \leq \int_{\tilde{\Omega}}\left|\operatorname{grad}\left(\tilde{\mathbf{u}}-\tilde{\wp}_{1, K, N}^{\mathrm{div}} \tilde{\mathbf{u}}\right)\right|^{2} R d R d \theta d Z
$$

hence there exists a positive constant $c$ independent of $\tilde{u}, K$ and $N$ such that

$$
\left\|\tilde{\mathbf{u}}_{K, N}-\tilde{\wp}_{1, K, N}^{\operatorname{div}} \tilde{\mathbf{u}}\right\|_{1, \#} \leq c\left\|\tilde{\mathbf{u}}-\tilde{\varphi}_{1, K, N}^{\operatorname{div}} \tilde{\mathbf{u}}\right\|_{1, \#} .
$$

Using the triangular inequality, we deduce

$$
\left\|\tilde{\mathbf{u}}-\tilde{\mathbf{u}}_{K, N}\right\|_{1, \#} \leq(1+c)\left\|\tilde{\mathbf{u}}-\tilde{\wp}_{1, K, N}^{\operatorname{div}} \tilde{\mathbf{u}}\right\|_{1, \#},
$$

and by using Theorem 3.4, we obtain (4.3). 
Appendix. The proof of the estimate (3.3) requires an interpolation result that we state in this appendix. To simplify the notations, for any real number $s$ with $s \geq 1$ we set

$$
\mathbf{H}_{\#, \rho}^{\circ s}(\Omega)=\mathbf{H}_{\#, \rho}^{s}(\Omega) \cap \mathbf{H}_{\#, \rho, 0}^{1}(\Omega)
$$

and

$$
\mathbf{H}_{\#, \rho}^{\circ s, \operatorname{div}}(\Omega)=\left\{\mathbf{u} \in\left(\mathbf{H}_{\#, \rho}^{\circ s}(\Omega)\right)^{3} ; \operatorname{div} \mathbf{u}=0\right\}
$$

We want to prove the following theorem.

THEOREM A.1. For any real numbers $\sigma, r$ and $\theta$ with $1 \leq r<\sigma$ and $0<\theta<1$, we have

$$
\left[\mathbf{H}_{\#, \rho}^{\circ \sigma, \operatorname{div}}(\Omega), \mathbf{H}_{\#, \rho}^{\circ r, \operatorname{div}}(\Omega)\right]_{\theta}=\mathbf{H}_{\#, \rho}^{\circ(1-\theta) \sigma+\theta r, \operatorname{div}}(\Omega) .
$$

For the notations of interpolated spaces we refer to Lions and Magenes [15]. This theorem is valid when $\rho$ is either the Legendre weight or the Chebyshev weight. We give its proof for the Chebyshev case.

We first recall an interpolation result for the spaces $\mathbf{H}_{\#, \rho}^{\circ s}(\Omega)$.

THEOREM A.2. For any real numbers $\sigma, r$ and $\theta$ with $1 \leq r<\sigma$ and $0<\theta<1$, we have

$$
\left[\left(\mathbf{H}_{\#, \rho}^{\circ \sigma}(\Omega)\right)^{3},\left(\mathbf{H}_{\#, \rho}^{\circ r}(\Omega)\right)^{3}\right]_{\theta}=\left(\mathbf{H}_{\#, \rho}^{\circ}(1-\theta) \sigma+\theta r(\Omega)\right)^{3} .
$$

This result has been proved by Y. Maday [18, Theorem 11.5] for the spaces $\left(\mathbf{H}_{\omega}^{\circ \sigma}\left(\Lambda^{3}\right)\right)^{3}$; the same proof gives (A.2) for the periodic/nonperiodic spaces $\left(\mathbf{H}_{\#, \rho}^{\circ \sigma}(\Omega)\right)^{3}$.

We first prove several lemmas. We find it convenient to introduce the following notations: for any real number $\lambda$ with $\lambda \neq 0$, set

$$
\left(\begin{array}{l}
\alpha_{\lambda}(z)=\operatorname{sh}(\lambda z) \\
\beta_{\lambda}(z)=\operatorname{ch}(\lambda z) \\
g_{\lambda}=-\lambda\left(\frac{\operatorname{ch}(\lambda)}{\left\|\alpha_{\lambda}\right\|_{0}^{2}} \alpha_{\lambda}+\frac{\operatorname{sh}(\lambda)}{\left\|\beta_{\lambda}\right\|_{0}^{2}} \beta_{\lambda}\right) .
\end{array}\right.
$$

LEMMA A.1. For any real number $\lambda$ with $\lambda \neq 0$, the following estimates hold:

$$
\begin{gathered}
\left\|\alpha_{\lambda}\right\|_{0, \rho} \leq\left\|\beta_{\lambda}\right\|_{0, \rho} \leq C_{2} \lambda^{-1 / 4} e^{\lambda}, \\
C_{1} \lambda^{-1 / 2} e^{\lambda} \leq\left\|\alpha_{\lambda}\right\|_{0} \leq\left\|\beta_{\lambda}\right\|_{0} \leq C_{2} \lambda^{-1 / 2} e^{\lambda},
\end{gathered}
$$

and

$$
\left\|\alpha_{\lambda}\right\|_{0, \rho^{-1}} \leq\left\|\beta_{\lambda}\right\|_{0, \rho^{-1}} \leq C_{2} \lambda^{-3 / 4} e^{\lambda}
$$

where $C_{1}$ and $C_{2}$ are positive constants independent of $\lambda$.

Proof. Setting $I_{j}=\int_{0}^{\pi / 2} \cos ^{2 j}(\theta) d \theta$, we recall that

$$
I_{j}-I_{j+1}=I_{j} /(2 j+2) \text { and } I_{j} \sim\{\pi /(4 j)\}^{1 / 2}
$$

hence there exists a positive constant $A$ such that

$$
\forall j \in \mathbb{N}, \quad\left(\begin{array}{l}
0 \leq I_{j} \leq A(2 j+1)^{-1 / 2} \\
0 \leq I_{j}-I_{j+1} \leq A(2 j+2)^{-1 / 2}(2 j+1)^{-1}
\end{array}\right.
$$

It is clear that

$$
\left\|\alpha_{\lambda}\right\|_{0, \rho} \leq\left\|\beta_{\lambda}\right\|_{0, \rho}, \quad\left\|\alpha_{\lambda}\right\|_{0, \rho^{-1}} \leq\left\|\beta_{\lambda}\right\|_{0, \rho^{-1}}
$$


and a direct calculation gives (A.5). To obtain (A.4) and (A.6), we note that

$$
\left\|\beta_{\lambda}\right\|_{0, \rho^{ \pm 1}}^{2}=\int_{0}^{1} 2[\operatorname{ch}(\lambda z)]^{2}[\rho(z)]^{ \pm 1} d z=\int_{0}^{1}[1+\operatorname{ch}(2 \lambda z)][\rho(z)]^{ \pm 1} d z
$$

and

$$
\int_{0}^{1} \operatorname{ch}(2 \lambda z)[\rho(z)]^{ \pm 1} d z=\sum_{j=0}^{\infty} \frac{(2 \lambda)^{2 j}}{(2 j) !} \int_{0}^{1} z^{2 j}[\rho(z)]^{ \pm 1} d z
$$

Using (A.7), we get

$$
\int_{0}^{1} z^{2 j} \rho(z) d z=I_{j} \leq A(2 j+1)^{-1 / 2}
$$

and

$$
\int_{0}^{1} z^{2 j}[\rho(z)]^{-1} d z=I_{j}-I_{j+1} \leq A(2 j+2)^{-1 / 2}(2 j+1)^{-1}
$$

hence we deduce

$$
\begin{aligned}
\int_{0}^{1} \operatorname{ch}(2 \lambda z) \rho(z) d z & \leq A \sum_{j=0}^{\infty} \frac{(2 \lambda)^{2 j}}{(2 j) !(2 j+1)^{1 / 2}} \\
& \leq A\left(\sum_{j=0}^{\infty} \frac{(2 \lambda)^{2 j}}{(2 j) !}\right)^{1 / 2}\left(\sum_{j=0}^{\infty} \frac{(2 \lambda)^{2 j}}{(2 j+1) !}\right)^{1 / 2} \\
& \leq A[\operatorname{ch}(2 \lambda) \operatorname{sh}(2 \lambda) / \lambda]^{1 / 2}
\end{aligned}
$$

This proves (A.4). We also have

$$
\begin{aligned}
\int_{0}^{1} \operatorname{ch}(2 \lambda z)[\rho(z)]^{-1} d z & \leq A \sum_{j=0}^{\infty} \frac{(2 \lambda)^{2 j}}{(2 j+1) !(2 j+2)^{1 / 2}} \\
& \leq A\left(\sum_{j=0}^{\infty} \frac{(2 \lambda)^{2 j}}{(2 j+1) !}\right)^{1 / 2}\left(\sum_{j=0}^{\infty} \frac{(2 \lambda)^{2 j}}{(2 j+2) !}\right)^{1 / 2} \\
& \leq A\left[\operatorname{sh}(2 \lambda) \operatorname{ch}(2 \lambda) /\left(8 \lambda^{3}\right)\right]^{1 / 2}
\end{aligned}
$$

which gives (A.6).

LEMMA A.2. For any real number $\lambda$ with $\lambda \geq 1$, and for $\varepsilon= \pm 1$, the solution $\varsigma_{\lambda, \varepsilon}$ of the problem

$$
\left(\begin{array}{l}
\left(\varsigma_{\lambda, \varepsilon}\right)^{\prime \prime}-\lambda^{2} \varsigma_{\lambda, \varepsilon}=g_{\lambda} \quad \text { in } \Lambda \\
\varsigma_{\lambda, \varepsilon}(1)=(1+\varepsilon) / 2, \quad \zeta_{\lambda, \varepsilon}(-1)=(1-\varepsilon) / 2
\end{array}\right.
$$

satisfies

$$
\varsigma_{\lambda, \varepsilon}^{\prime}(-1)=\varsigma_{\lambda, \varepsilon}^{\prime}(1)=0
$$

and

$$
\forall j \in \mathbb{N}, \quad\left\|\zeta_{\lambda, \varepsilon}\right\|_{j, \rho} \leq C_{j} \lambda^{j-1 / 4}
$$

where $C_{j}$ are positive constants independent of $\lambda$.

Proof. We prove the lemma for $\varepsilon=1$; the case $\varepsilon=-1$ is analogous. We first remark that if $\varphi$ is such that $\varphi^{\prime \prime}-\lambda^{2} \varphi=0$ we have

$$
\left(\varsigma_{\lambda, 1}^{\prime} \varphi-\varsigma_{\lambda, 1} \varphi^{\prime}\right)^{\prime}=g_{\lambda} \varphi
$$


and then

$$
\varsigma_{\lambda, 1}^{\prime}(1) \varphi(1)-\varsigma_{\lambda, 1}^{\prime}(-1) \varphi(-1)=\varphi^{\prime}(1)+\int_{-1}^{1} g_{\lambda}(t) \varphi(t) d t .
$$

Applying this relation with $\varphi=\alpha_{\lambda}$ and $\varphi=\beta_{\lambda}$, we obtain

$$
\varsigma_{\lambda, 1}^{\prime}(1)+\varsigma_{\lambda, 1}^{\prime}(-1)=\varsigma_{\lambda, 1}^{\prime}(1)-\varsigma_{\lambda, 1}^{\prime}(-1)=0,
$$

and this gives (A.8). Then $S_{\lambda, 1}^{\prime}$ is the solution of the problem

$$
\left(\begin{array}{l}
\left(\varsigma_{\lambda, 1}^{\prime}\right)^{\prime \prime}-\lambda^{2} \varsigma_{\lambda, 1}^{\prime}=g_{\lambda}^{\prime} \\
\varsigma_{\lambda, 1}^{\prime}(1)=\varsigma_{\lambda, 1}^{\prime}(-1)=0
\end{array}\right.
$$

hence we obtain the classical estimates

$$
\left\|\zeta_{\lambda, \varepsilon}^{\prime}\right\|_{0, \rho} \leq C \lambda^{-2}\left\|g_{\lambda}^{\prime}\right\|_{0, \rho}, \quad\left\|\zeta_{\lambda, \varepsilon}^{\prime \prime}\right\|_{0, \rho} \leq C \lambda^{-1}\left\|g_{\lambda}^{\prime}\right\|_{0, \rho}
$$

On the other hand,

$$
\left\|g_{\lambda}\right\|_{0, \rho} \leq \lambda\left\{\operatorname{ch}(\lambda) \frac{\left\|\alpha_{\lambda}\right\|_{0, \rho}}{\left\|\alpha_{\lambda}\right\|_{0}^{2}}+\operatorname{sh}(\lambda) \frac{\left\|\beta_{\lambda}\right\|_{0, \rho}}{\left\|\beta_{\lambda}\right\|_{0}^{2}}\right\}
$$

and

$$
\left\|g_{\lambda}^{\prime}\right\|_{0, \rho} \leq \lambda^{2}\left\{\operatorname{ch}(\lambda) \frac{\left\|\beta_{\lambda}\right\|_{0, \rho}}{\left\|\alpha_{\lambda}\right\|_{0}^{2}}+\operatorname{sh}(\lambda) \frac{\left\|\alpha_{\lambda}\right\|_{0, \rho}}{\left\|\beta_{\lambda}\right\|_{0}^{2}}\right\} .
$$

Using (A.4) and (A.5), we deduce $\left\|g_{\lambda}\right\|_{0, \rho} \leq C \lambda^{7 / 4}$ and $\left\|g_{\lambda}^{\prime}\right\|_{0, \rho} \leq C \lambda^{11 / 4}$; hence from (A.10) we get

$$
\left\|\zeta_{\lambda, \varepsilon}^{\prime}\right\|_{0, \rho} \leq C \lambda^{3 / 4}, \quad\left\|\zeta_{\lambda, \varepsilon}^{\prime \prime}\right\|_{0, \rho} \leq C \lambda^{7 / 4} .
$$

Using now the inequality

$$
\left\|\zeta_{\lambda, \varepsilon}\right\|_{0, \rho} \leq \lambda^{-2}\left\{\left\|g_{\lambda}\right\|_{0, \rho}+\left\|\zeta_{\lambda, \varepsilon}^{\prime \prime}\right\|_{0, \rho}\right\}
$$

we obtain

$$
\left\|\zeta_{\lambda, \varepsilon}\right\|_{0, \rho} \leq C \lambda^{-1 / 4}
$$

By virtue of (A.11) and (A.12) we have (A.9) for $j \in\{0,1,2\}$. By using the equality $\varsigma_{\lambda}^{\prime \prime}=\lambda^{2} \varsigma_{\lambda}+g_{\lambda}$ we finally obtain (A.9) for $j \geq 3$ by induction.

LEMMA A.3. For any real number $\lambda$ with $\lambda \neq 0$ and any function $f$ in $\mathrm{L}_{\rho}^{2}(\Lambda)$, the solution $\varphi$ of the problem

$$
\left(\begin{array}{l}
\varphi^{\prime \prime}-\lambda^{2} \varphi=f \quad \text { in } \Lambda \\
\varphi(1)=\varphi(-1)=0
\end{array}\right.
$$

satisfies

$$
\left|\varphi^{\prime}(-1)\right|+\left|\varphi^{\prime}(1)\right| \leq C \lambda^{-3 / 4}\|f\|_{0, \rho},
$$

where $C$ is a positive constant independent of $\lambda, \varphi$ and $f$.

Proof. Setting

$$
\chi(z)=\frac{\operatorname{sh}(\lambda\{z+1\})}{\operatorname{sh}(2 \lambda)}
$$

we have the relation $\left(\varphi^{\prime} \chi-\varphi \chi^{\prime}\right)^{\prime}=f \chi$; hence

$$
\varphi^{\prime}(1)=\int_{-1}^{1} f(z) \chi(z) d z \text {. }
$$


This gives the estimate

$$
\left|\varphi^{\prime}(1)\right| \leq\|f\|_{0, \rho}\|\chi\|_{0, \rho^{-1}}
$$

On the other hand,

$$
\chi=\frac{\operatorname{ch}(\lambda) \alpha_{\lambda}+\operatorname{sh}(\lambda) \beta_{\lambda}}{\operatorname{sh}(2 \lambda)}
$$

and from (A.6) and (A.14) we get

$$
\left|\varphi^{\prime}(1)\right| \leq C \lambda^{-3 / 4}\|f\|_{0, \rho}
$$

In the same way we obtain $\left|\varphi^{\prime}(-1)\right| \leq C \lambda^{-3 / 4}\|f\|_{0, \rho}$.

We are now in a position to state the following theorem.

THEOREM A.3. Let $r$ be a nonnegative real number. For any function $p$ in $\mathbf{H}_{\#, \rho}^{r}(\Omega)$ with $\int_{\Omega} p(\mathbf{x}) d \mathbf{x}=0$, there exists a function $\mathbf{u}$ in $\left(\mathbf{H}_{\#, \rho}^{r+1}(\Omega)\right)^{3} \cap\left(\mathbf{H}_{\#, \rho, 0}^{1}(\Omega)\right)^{3}$ such that

$$
\operatorname{div} \mathbf{u}=p
$$

and

$$
\|\mathbf{u}\|_{r+1, \#, \rho} \leq C_{r}\|p\|_{r, \#, \rho}
$$

where $C_{r}$ is a positive constant independent of $p$.

Proof. We prove (A.16) when $r$ is an integer. The general case will follow by using an interpolation argument.

We look for $\mathbf{u}$ in the form $\mathbf{u}=\operatorname{grad} \varphi+\mathbf{w}$, where $\varphi$ is the solution of the problem

$$
\left(\begin{array}{l}
\Delta \varphi=p \quad \text { in } \Omega, \\
\varphi \in \mathbf{H}_{\#, \rho, 0}^{1}(\Omega) .
\end{array}\right.
$$

To this end we write $p$ and $\varphi$ in the form

$$
\begin{aligned}
& p(\mathbf{x})=\sum_{\mathbf{k} \in \mathbb{Z}^{2}} p_{\mathbf{k}}(z) e^{i\left(k_{1} x+k_{2} y\right)}, \quad \text { with } \int_{-1}^{1} p_{0}(z) d z=0 \\
& \varphi(\mathbf{x})=\sum_{\mathbf{k} \in \mathbf{Z}^{2}} \varphi_{\mathbf{k}}(z) e^{i\left(k_{1} x+k_{2} y\right)}
\end{aligned}
$$

For any $\mathbf{k}$ in $\mathbb{Z}^{2}, \varphi_{\mathbf{k}}$ is the solution of the problem

$$
\left(\begin{array}{l}
\left(\varphi_{\mathbf{k}}\right)^{\prime \prime}-|\mathbf{k}|_{2}^{2} \varphi_{\mathbf{k}}=p_{\mathbf{k}} \\
\varphi_{\mathbf{k}}(-1)=\varphi_{\mathbf{k}}(1)=0
\end{array}\right.
$$

Since $\int_{-1}^{1} p_{0}(z) d z=0$, we have $\int_{-1}^{1}\left(\varphi_{0}\right)^{\prime \prime}(z) d z=0$, hence

$$
\varphi_{0}^{\prime}(-1)=\varphi_{0}^{\prime}(1)
$$

Moreover,

$$
\left|\varphi_{0}^{\prime}(-1)\right|+\left|\varphi_{0}^{\prime}(1)\right| \leq C\left\|\varphi_{0}\right\|_{2, \rho} \leq C\left\|p_{0}\right\|_{0, \rho} .
$$

Using (A.13), we also get

$$
\forall \mathbf{k} \in \mathbb{Z}^{2}, \mathbf{k} \neq \mathbf{0}, \quad\left|\varphi_{\mathbf{k}}^{\prime}(-1)\right|+\left|\varphi_{\mathbf{k}}^{\prime}(1)\right| \leq C|\mathbf{k}|_{2}^{-3 / 4}\left\|p_{\mathbf{k}}\right\|_{0, \rho} .
$$

We now set

$$
\mathbf{v}=\operatorname{grad} \varphi=\left(v_{1}, v_{2}, v_{3}\right)
$$


It is easy to show that (see, e.g., [7, Lemma 11.3])

Moreover,

$$
\left(\begin{array}{l}
\|\mathbf{v}\|_{r+1, \#, \rho} \leq C\|p\|_{r, \#, \rho} \\
\operatorname{div} \mathbf{v}=p \\
\left(v_{1}, v_{2}\right) \in\left(\mathbf{H}_{\#, \rho, 0}^{1}(\Omega)\right)^{2}
\end{array}\right.
$$

$$
v_{3}(\mathbf{x})=\sum_{\mathbf{k} \in \mathbf{Z}^{2}} \varphi_{\mathbf{k}}^{\prime}(z) e^{i\left(k_{1} x+k_{2} y\right)} .
$$

We now define the divergence-free function $\mathbf{w}$ as follows:

$$
\mathbf{w}(\mathbf{x})=\sum_{\mathbf{k} \in \mathbb{Z}^{2}} w_{\mathbf{k}}(z) e^{i\left(k_{1} x+k_{2} y\right)},
$$

where the functions $w_{\mathbf{k}}$ are defined as follows:

(1)

$$
w_{0}(z)=\left(\begin{array}{c}
0 \\
0 \\
-\varphi_{0}^{\prime}(1)
\end{array}\right) \text {. }
$$

(2) For any $\mathbf{k}$ in $\mathbb{Z}^{2}$ with $\mathbf{k} \neq \mathbf{0}$, we set

$$
\psi_{\mathbf{k}}=\varphi_{\mathbf{k}}^{\prime}(1)_{\zeta|\mathbf{k}|_{2}, 1}+\left.\varphi_{\mathbf{k}}^{\prime}(-1) \zeta_{\mid \mathbf{k}}\right|_{2,-1} ^{\prime}
$$

(where $\zeta_{\lambda, \varepsilon}$ is defined in Lemma A.2) and

$$
w_{\mathbf{k}}(z)=|\mathbf{k}|_{2}^{-2}\left(\begin{array}{c}
-i k_{1} \psi_{\mathbf{k}}^{\prime}(z) \\
-i k_{2} \psi_{\mathbf{k}}^{\prime}(z) \\
-|\mathbf{k}|_{2}^{2} \psi_{\mathbf{k}}(z)
\end{array}\right) .
$$

Using (A.17) and Lemma A.2, we have

$$
\mathbf{v}+\mathbf{w} \in\left(\mathbf{H}_{\#, \rho, 0}^{1}(\Omega)\right)^{3} .
$$

Finally, using (A.7), we deduce

$$
\begin{aligned}
\|\mathbf{w}\|_{r+1, \#, \rho}^{2} \leq\left|\varphi_{0}^{\prime}(1)\right|^{2}+\sum_{\substack{\mathbf{k} \in \mathbf{Z}^{2} \\
\mathbf{k} \neq 0}}\left\{|\mathbf{k}|_{2}^{2 r+2}\left\|\psi_{\mathbf{k}}\right\|_{0, \#, \rho}^{2}+|\mathbf{k}|_{2}^{2 r}\left\|\psi_{\mathbf{k}}\right\|_{1, \#, \rho}^{2}\right. \\
\left.+\left\|\psi_{\mathbf{k}}\right\|_{r+1, \#, \rho}^{2}+|\mathbf{k}|_{2}^{-2}\left\|\psi_{\mathbf{k}}\right\|_{r+2, \#, \rho}^{2}\right\} .
\end{aligned}
$$

Using (A.9), (A.19) and (A.21), we obtain the estimates

$$
\forall j \in \mathbb{N}, \quad\left\|\psi_{\mathbf{k}}\right\|_{j, \#, \rho} \leq C|\mathbf{k}|_{2}^{j-1}\left\|p_{\mathbf{k}}\right\|_{0, \#, \rho},
$$

which, using (A.18) and (A.22), give the estimate

$$
\|\mathbf{w}\|_{r+1, \#, \rho} \leq C\|p\|_{r, \#, \rho} .
$$

Theorem A.3 is then proved with $\mathbf{u}=\mathbf{v}+\mathbf{w}$.

Proof of Theorem A.1. Theorem A.1 follows from Theorems A.2 and A.3, using [15, Vol. 1, Theorem 14.3].

Istituto Analisi Numerica del C.N.R.

Corso C. Alberto, 5

27100 Pavia, Italy

Analyse Numérique

Université Pierre et Marie Curie

4, place Jussieu

F-75230 Paris Cedex 05, France 
1. R. A. AdAmS, Sobolev Spaces, Academic Press, New York, 1975.

2. A. Avantaggiati, "Spazi di Sobolev con peso ed alcune applicazioni," Boll. Un. Mat. Ital., v. 5,1976 , pp. 1-52.

3. I. BABUŠKA, B. A. SZABO \& I. N. KATZ, The p-Version of the Finite Element Method, Report of the Washington University, St. Louis, 1979.

4. J. Bergh \& J. Lofstrom, Interpolation Spaces. An Introduction, Springer-Verlag, Berlin and New York, 1976.

5. C. BERnARDI, C. CANUTO \& Y. MADAY, "Generalized inf-sup condition for Chebyshev approximation of the Navier-Stokes equations," SIAM J. Numer. Anal., v. 25, 1988.

6. C. BERNARDI \& Y. MADAY, "Properties of some weighted Sobolev spaces, An application to spectral approximation," SIAM J. Numer. Anal. (To appear.)

7. C. BERNARDI, Y. MADAY \& B. MÉTIVET, "Spectral approximation of the periodic nonperiodic Navier-Stokes equations," Numer. Math., v. 51, 1987, pp. 655-700.

8. C. Bernardi, Y. MADAY \& B. MÉtivet, "Calcul de la pression dans la résolution spectrale du problème de Stokes," Rech. Aérospat., v. 1, 1986, pp. 1-21.

9. C. CANUTO \& A. QUARTERONI, "Spectral and pseudo-spectral methods for parabolic problems with nonperiodic boundary conditions," Calcolo, v. 18, 1981, pp. 197-218.

10. C. CANUTO \& A. QUARTERONI, "Approximation results for orthogonal polynomials in Sobolev spaces," Math. Comp., v. 38, 1982, pp. 67-86.

11. C. Canuto \& G. SaCchi LANDRiani, "Analysis of the Kleiser-Schumann method," $\mathrm{Nu}$ mer. Math., v. 50, 1986, pp. 217-243.

12. P. GRISVARD, "Espaces intermédiaires de Sobolev avec poids," Ann. Scuola Norm. Sup. Pisa, v. 17, 1963, pp. 255-296.

13. L. KLEISER \& U. SCHUMANN, "Treatment of incompressibility and boundary conditions in 3-D numerical spectral simulations of plane channel flows," in Proc. Third GAMM Conf. on Numer. Methods in Fluid Mech. (E. H. Hirschel, ed.), Vieweg (Braunschweig), 1980, pp. 165-173.

14. H.-O. KREISS \& J. Oliger, "Stability of the Fourier method," SIAM J. Numer. Anal., v. 16, 1979, pp. 421-433.

15. J. L. Lions \& E. MAgenes, Non Homogeneous Boundary Value Problems and Applications, Vols. 1 and 2, Springer-Verlag, Berlin and New York, 1972.

16. N. MAC GIOlla MHUIRIS, The Construction and Use of Divergence-Free Vector Expansions for Incompressible Fluid Flow Calculation, ICASE Report no. 86-20, 1986.

17. Y. MADAY, "Analysis of spectral projectors in one-dimensional domains," Math. Comp. (To appear.)

18. Y. MADAY, "Analysis of spectral projectors in multi-dimensional domains," submitted for publication .

19. Y. MADAY \& B. MÉTIVET, "Chebyshev spectral approximation of Navier-Stokes equations in a two-dimensional domain," $M^{2} A N$, v. 21, 1987, pp. 93-123.

20. Y. MADAY \& A. QUARTERONI, "Legendre and Chebyshev spectral approximations of Burgers' equation," Numer. Math., v. 37, 1981, pp. 321-332.

21. R. D. MOSER, P. MOIN \& A. LEONARD, "A spectral numerical method for the NavierStokes equations with applications to Taylor-Couette flow," J. Comput. Phys., v. 52, 1983, pp. 524-544.

22. J. PASCIAK, "Spectral and pseudospectral methods for advection equations," Math. Comp., v. 35, 1980, pp. 1081-1092.

23. F. PASQUARElli, A. QUARTERONi \& G. SACChi LANDRIANi, "Spectral approximations of the Stokes problem by divergence-free functions," J. Sci. Comput., v. 2, 1987, pp. 195-226.

24. G. SACCHI LANDRIANI, "Convergence of the Kleiser-Schumann method for Navier-Stokes equations," Calcolo, v. 23, 1986, pp. 383-406.

25. G. SACCHI LANDRIANI, "Spectral tau approximation of the two-dimensional Stokes problem," Numer. Math., v. 52, 1988, pp. 683-699.

26. G. SACCHI LANDRIANI \& H. VANDEVEN, "Approximation polynômiale de fonctions à divergence nulle," C. R. Acad. Sci. Paris Sér. I, v. 304, no. 3, 1987. 